\title{
Leber und Gallenwege
}

Markus Paschold, Hauke Lang

$21.1 \quad$ Akute Gallenblasenkolik - 273

21.1.1 Klinik und Diagnostik - 273

21.1.2 Therapie -273

21.2 Akute Cholezystitis - 274

21.2.1 Klinik und Diagnostik - 274

21.2.2 Therapie - 275

21.3 Gallenblasenperforation - 275

21.3.1 Klinik und Diagnostik - 276

21.3.2 Therapie -276

21.4 Cholangiolithiasis - 276

21.4.1 Klinik und Diagnostik - 276

21.4.2 Therapie -277

21.5 Verschlussikterus -277

21.5.1 Klinik und Diagnostik - 278

21.5.2 Therapie -278

21.6 Cholangitis -278

21.6.1 Klinik und Diagnostik - 278

21.6.2 Therapie -279

21.7 Benigne Gallengangsstrikturen - 279

21.7.1 Klinik und Diagnostik - 280

21.7.2 Therapie -280

21.8 Portale Hypertension - 280

21.8.1 Klinik und Diagnostik - 280

21.8.2 Therapie -281

21.9 Pyogener Leberabszess - 283

21.9.1 Klinik und Diagnostik - 284

21.9.2 Therapie -284 
21.10 Leberversagen - 284

21.10.1 Klinik und Diagnostik - 285

21.10.2 Therapie - 285

21.10.3 Praktische Hinweise - 286

21.11 Komplikationen nach ERCP und interventionellen

Eingriffen - 287

21.12 Postoperative Komplikationen - 288

21.12.1 Biliäre Leckage - 288

21.12.2 Biliäre Strikturen - 289

21.12.3 Blutungen -289

21.12.4 Cholangitis - 289

21.12.5 Abszess - 289

21.12.6 Biliom - 290

Literatur -290 


\subsection{Akute Gallenblasenkolik}

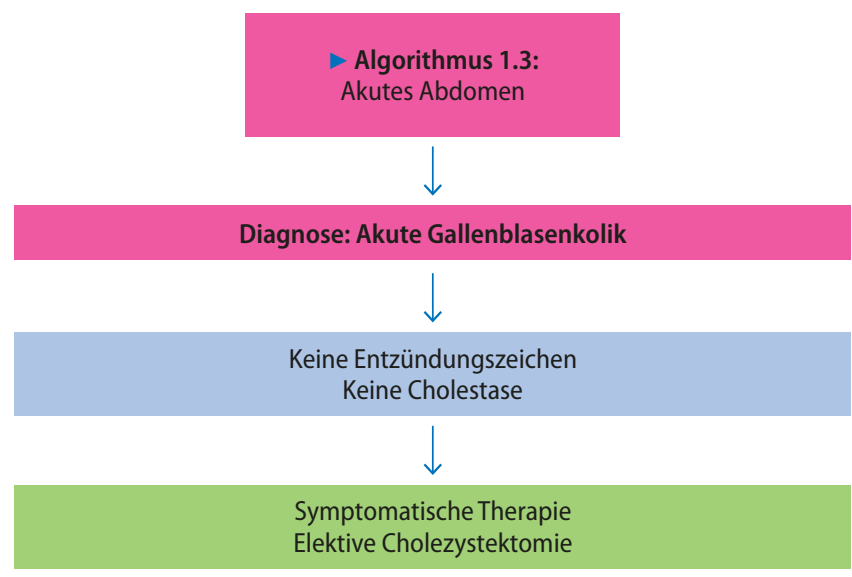

Patienten mit einer akuten Gallenblasenkolik müssen im Verlauf mit erneuten Beschwerden oder Komplikationen rechnen (siehe Übersicht). Sie sollten daher so bald wie möglich einer Cholezystektomie zugeführt werden, weil sich dadurch die Morbidität während der Wartezeit für eine elektive Cholezystektomie und wiederholte notfallmäßige Vorstellungen in der Notaufnahme vermeiden lassen. Die frühe laparoskopische Cholezystektomie scheint dabei mit einer niedrigeren Konversionsrate, einer kürzeren Operationszeit und einem kürzeren Krankenhausaufenthalt einherzugehen (Duncan u. Riall 2013).

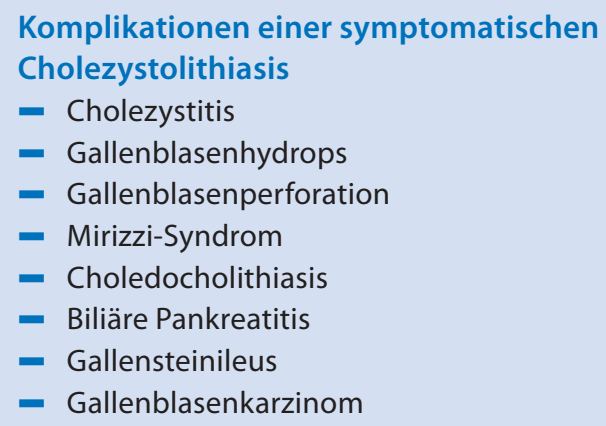

\subsubsection{Klinik und Diagnostik}

Anfallsartige, an Intensität wechselnde kolikartige Schmerzen im rechten Oberbauch mit Ausstrahlung in den Rücken und die rechte Schulter, begleitet von Allgemeinsymptomen, sind das typische Leitsymptom der akuten Gallenblasenkolik. Fieber und eine Leukozytose fehlen in den meisten Fällen. Das Auftreten einer Cholestase gefolgt von einem flüchtigen Ikterus nach initialer Kolik sind Zeichen eines Steinabganges in den Ductus choledochus (DHC). Anamnese, klinische Untersuchung, labormedizinische Untersuchungen (alkalische Phosphatase [AP], $\gamma$-GT, Bilirubin, Aspartat-Aminotransferase [AST], Alanin-Aminotransferase [ALT], Blutbild, C-reaktives Protein [CRP], Pankreasenzyme) und Oberbauchsonographie führen in über $95 \%$ der Fälle zur Diagnose.

\subsubsection{Therapie}

Neben Bettruhe und Nahrungskarenz sollten Analgetika (cave: kein Morphin) und Spasmolytika während der akuten Gallenblasenkolik verabreicht werden. Die laparoskopische Cholezystektomie ist das Standardverfahren, die wegen der oben genannten Gründe frühelektiv durchgeführt werden sollte. Die Vorteile gegenüber der konventionellen Cholezystektomie sowie das operative Vorgehen sind hinlänglich bekannt. Die möglichen Komplikationen einer Cholezystektomie sind in der u. g. Übersicht aufgelistet. Die Letalität beträgt unter Berücksichtigung der Patientenselektion und Kontraindikationen (Verdacht auf Gallenblasen- oder Gallengangkarzinom, große abdominale Voroperationen, ausgeprägte portale Hypertension wegen einer Leberzirrhose, hohes kardiopulmonales Risiko) $<0,1 \%$.

Bei nicht beherrschbaren intraoperativen Blutungen, unübersichtlichen anatomischen Verhältnissen oder Verdacht auf ein Gallenblasenkarzinom ist der Umstieg auf die konventionelle Cholezystektomie nicht zu vermeiden (ca. 5 \%) und sollte rechtzeitig erfolgen. Verletzungen oder Durchtrennungen der Gallenwege entstehen in den meisten Fällen, wenn unübersichtliche anatomische Verhältnisse vorliegen. Daher empfehlen wir die Durchführung einer intraoperativen Cholangiographie bei unklaren anatomischen Verhältnissen. Eine zu tiefe Präparationsebene in das Leberparenchym hinein kann zu einer Blutung führen. Weitere Blutungsursachen sind eine Verletzung der A. cystica, ein Abgleiten der Ligatur bzw. der Endoclips und die Trokareinstichstellen. Das Management einer erheblichen Blutung während der laparoskopischen Cholezystektomie kann risikoreich und mit erheblichen Gefahren verbunden sein. Diese beinhalten Ligaturen oder Verletzungen größerer Gefäße und/oder der Gallenwege durch ungezielte Clipapplikationen oder Gebrauch des Elektrokauters in einem schlecht visualisierten Operationsfeld (- Abb. 21.1).

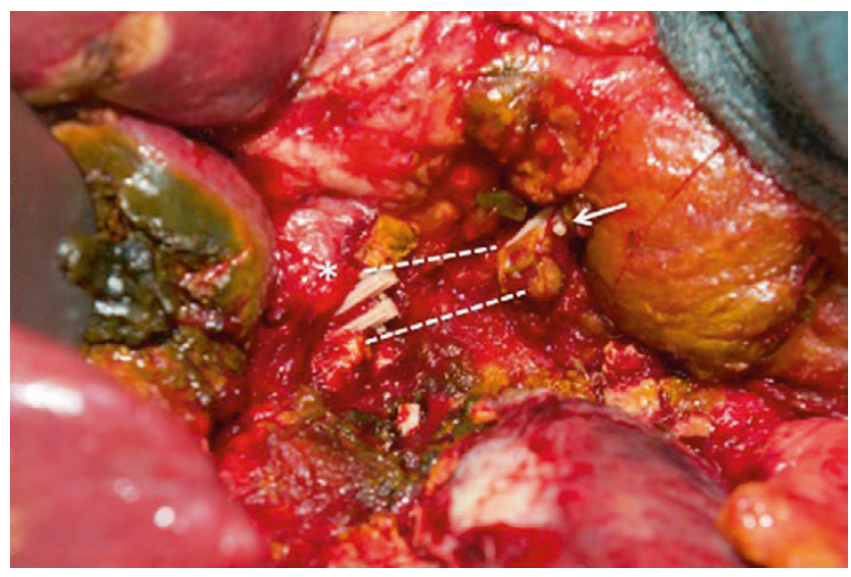

- Abb. 21.1 Im Rahmen einer laparoskopischen Cholezystektomie erfolgte eine akzidentelle Resektion des DHC mit der Gallenblase (gestrichelte Linie zeigt den ursprünglichen Verlauf des DHC). Der DHC wurde in Höhe der Hepatikusgabel (*) und supraduodenal (Pfeil) abgesetzt. Die rechte Leberarterie aus der A. mesenterica superior wurde dabei ebenfalls supraduodenal abgesetzt 
In solchen Fällen ist die Konversion zu einer Laparotomie das oftmals weitaus bessere Vorgehen, um eine gezielte Blutstillung zu erzielen und Verletzungen zu vermeiden.

Better safe than sorry!

Komplikationen der Cholezystektomie

- Zystikusstumpfinsuffizienz

- Ligatur, Verletzung oder Durchtrennung von DHC oder Ductus hepaticus
- Gallefistel (Verletzung oder Durchtrennung von DHC oder Ductus hepaticus, Eröffnung eines akzessorischen Gangs)

- Ligatur, Verletzung oder Durchtrennung der A. hepatica dextra

- Intraoperative Blutungen

- Gefäß- oder Darmverletzung beim Einbringen der Trokare

- "Postcholezystektomiesyndrom"

\subsection{Akute Cholezystitis}

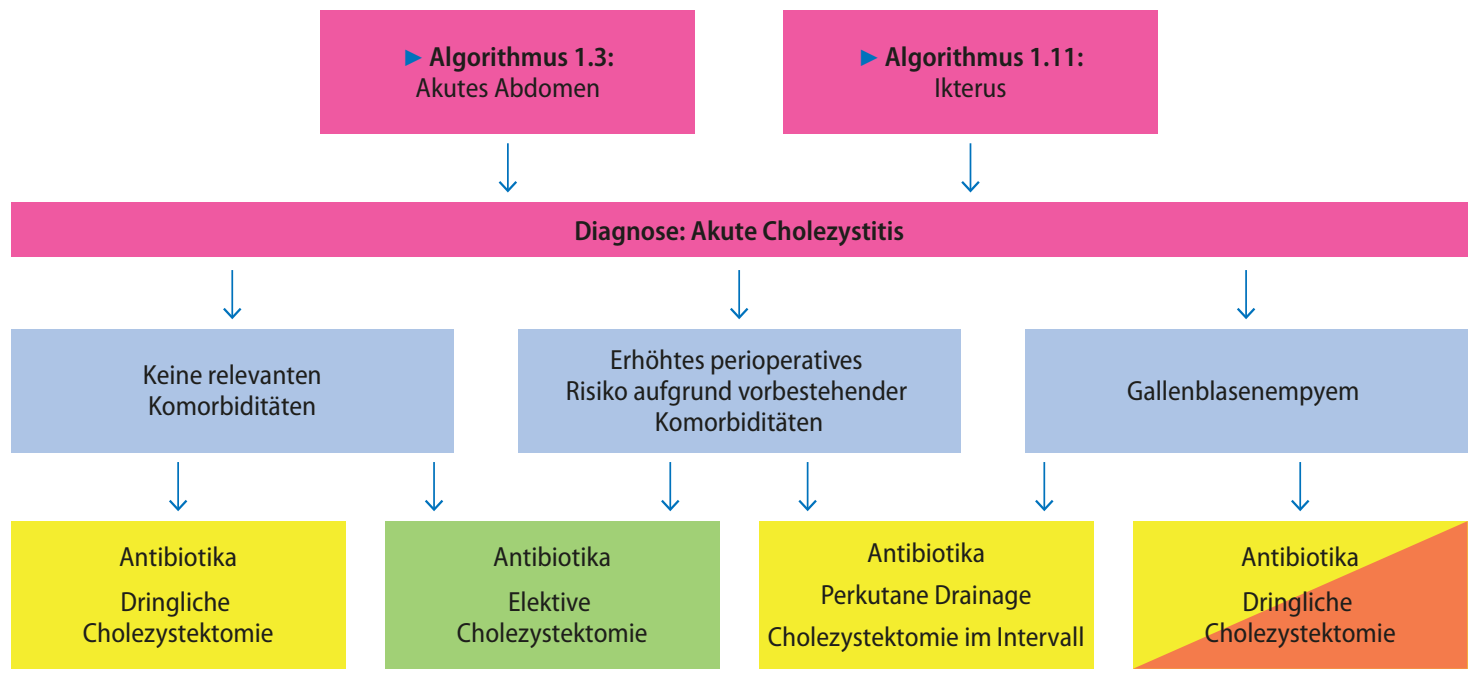

Die akute Cholezystitis ist die häufigste Komplikation der Cholezystolithiasis und erfordert eine notfallmäßige Behandlung. Die Ursache der akuten Cholezystitis ist ein impaktierter Gallenstein entweder im Infundibulum oder im Ductus cysticus. Es resultiert eine Distension der Gallenblase mit Ödem und akuter Entzündung der Gallenblasenwand, was zu einer venösen Stase und Obstruktion sowie zu einer Thrombose der Arteria (A.) cystica führen kann. In der Folge kommt es zu einer Ischämie oder Nekrose der Gallenblase, die konsekutiv zu einer phlegmonösen oder gangränösen Cholezystitis mit Perforationsgefahr führen können. Weitere Komplikationen sind ein Gallenblasenempyem durch einen superinfizierten Gallenblasenhydrops, eine emphysematöse Cholangitis durch gasbildende Bakterien in der Gallenflüssigkeit, das Mirizzi-Syndrom mit Cholestase, eine chronische Cholezystitis infolge einer Kompression des Ductus hepatocholedochus (DHC) durch einen Infundibulumstein, oder auch eine cholezystoenterische Fistel mit Steinabgang ins Intestinum und der Gefahr eines konsekutiven mechanischen Dünndarmileus (Gallensteinileus).

\subsubsection{Klinik und Diagnostik}

Das klinische Zeichen einer akuten Cholezystitis ist das Murphy-Zeichen. Bei tiefer Palpation im rechten Oberbauch kommt es reflektorisch zum Anhalten der Inspiration. Es zeigen sich konstant starke Schmerzen im rechten Oberbauch oder Epigastrium, welche in die rechte Schulter und rechte Flanke ausstrahlen können. Gallenkoliken, Übelkeit, Erbrechen und Inappetenz können diesem Schmerzereignis vorausgehen. Differenzialdiagnostisch muss an ein perforiertes Ulkus, eine Gastritis oder Gastroenteritis, chronischentzündliche Darmerkrankungen, eine rechte Unterlappenpneumonie oder eine Gallenwegsdyskinesie gedacht werden.

\section{Praxistipp}

Die Diagnose einer akuten Cholezystitis erfolgt schnell und sicher mit der Sonographie. Der Nachweis von Gallensteinen, eine Verdickung der Gallenblasenwand (>4 mm) und pericholezystitische Flüssigkeitsansammlung sind typische Befunde. 


\subsubsection{Therapie}

Die Behandlung erfordert eine sofortige parenterale Antibiotika- und Analgetikagabe in Vorbereitung auf eine Cholezystektomie, welche die definitive Therapie einer akuten Cholezystitis darstellt. Wir bevorzugen im eigenen Vorgehen wegen der im Falle einer Perforation und Peritonitis mit einem septischen Verlauf bestehenden Letalität von bis zu ca. 30 \% die sofortige oder zeitnahe Operation innerhalb von 24 Stunden. Die Operation wird standardmäßig als laparoskopische Cholezystektomie durchgeführt. Die Kosteneffektivität durch einen kürzeren Krankenhausaufenthalt und Vermeidung von Wiederaufnahmen wegen rezidivierender Cholezystitis oder Gallenkoliken sind Argumente für einen frühen Operationszeitpunkt. Die Patienten weisen bei diesem Vorgehen einen geringeren Schmerzmittelbedarf, kosmetisch günstigere Ergebnisse und eine niedrige Morbidität oder Mortalität auf.

Die behandlungsbedürftigen operativen Komplikationen sind insgesamt gering $(2,4 \%)$ und betreffen überwiegend Wundinfektionen, viel seltener die Ligatur der A. hepatica dextra, Choledochusverletzung, Gallefistel z. B. durch Verletzung eines akzessorischen Gallengangs oder Zystikusstumpfinsuffizienz, Pankreatitis, Blutung oder Nachblutungen sowie Verletzungen an Darm oder Blutgefäßen durch die Trokarapplikation. Bei einer akuten Cholezystitis beträgt die Konversionsrate zur konventionellen Cholezystektomie etwa $10 \%$. Die Konversion sollte bei einer schlechten Visualisierung der anatomischen Strukturen und Verhältnisse im Calot-Dreieck, einer vermuteten Gallengangsverletzung, laparoskopisch nicht beherrschbarer Blutung oder langer Operationszeit großzügig erfolgen.

\section{Praxistipp}

Im Falle schwerer entzündlicher Veränderungen, die eine Visualisierung der Strukturen im Calot-Dreieck erschweren oder gar unmöglich machen, empfehlen wir, die Dissektion vom Fundus der Gallenblase aus zu beginnen und erst im Verlauf den Gallenblasenhals und den Ductus cysticus darzustellen. In seltenen Fällen sollte nur eine subtotale Cholezystektomie erfolgen, um Verletzungen der Gallenwege oder der Hilusgefäße zu vermeiden. Der Fundus der Gallenblase wird hierbei eröffnet, der Gallenblaseninhalt evakuiert und die Vorderwand der Gallenblase exzidiert. Es verbleibt die im Gallenblasenbett der Leber anhängende Gallenblasenhinterwand. Der Versuch, den Zystikus zu ligieren, ist in dieser Situation gefährlich, prädisponiert zu Verletzungen von DHC oder Leberarterie und sollte unterbleiben, insbesondere wenn keine Gallefistel sichtbar ist.

Im Falle eines erhöhten perioperativen Risikos aufgrund vorbestehender Komorbiditäten - Alter $>65$ Jahre und ASA (American Society of Anesthesiologists Physical Status) $\geq 3$ - erfolgt zunächst eine konservative Therapie mit parenteraler Gabe von Antibiotika, Antiphlogistika und Spasmolytika so- wie ggf. eine perkutane Cholezystostomie. Eine Drainage sollte dann mittels Sonographie oder Computertomographie (CT) transhepatisch platziert werden. Sie entlastet die Gallenblase, evakuiert die infizierte Gallenflüssigkeit und lindert den durch die Gallenblasendistension hervorgerufenen Schmerz. Unter diesem Behandlungsregime verbessert sich in kurzer Zeit der klinische Zustand in über $80 \%$ aller Fälle. Die Cholezystektomie erfolgt, falls es die klinische Situation rechtfertigt, nach Stabilisierung des Patienten im Intervall von bis zu 6 Wochen.

10-30\% der Patienten mit einer akuten Cholezystitis entwickeln unter konservativer Therapie Komplikationen mit einer sehr ernsten Prognosen wie Gallenblasenempyem, gangränöse Cholezystitis, Gallenblasenperforation, emphysematöse Cholezystitis, Mirizzi-Syndrom und cholezystoenterische Fistel mit ggf. Entwicklung eines Gallensteinileus. In diesen Fällen ist neben der intravenösen Antibiotikagabe die notfallmäßige Cholezystektomie erforderlich, sofern es der klinische Zustand des Patienten zulässt. Alternativ können kritisch kranke Patienten auch mit einer temporären, perkutanen Drainage behandelt werden.

\subsection{Gallenblasenperforation}

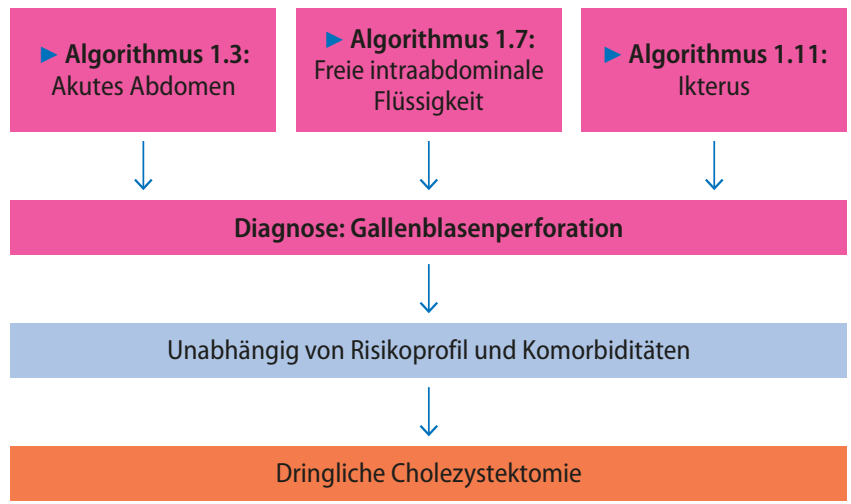

Die Gallenblasenperforation ist eine lebensbedrohliche Komplikation einer akuten Cholezystitis. Es resultiert eine Distension der Gallenblase mit Ödem der Gallenblasenwand, die zu einer venösen Stase und Obstruktion sowie einer Thrombose der A. cystica führen kann. In der Folge kommt es zu einer Ischämie oder Nekrose der Gallenblase mit konsekutiver phlegmonöser oder gangränöser Cholezystitis und Perforation.

Die Gallenblasenperforation in ein angrenzendes Hohlorgan ist eine seltene Komplikation. Infolge der Kontamination des intra- und extrahepatischen Gallengangsystems durch intestinale Mikroorganismen kann es zu einer aszendierenden Cholangitis kommen. Das Duodenum und das Colon transversum sind die häufigsten Orte einer cholezystoenterischen Fistel, und ein Steinabgang über diese Fistel birgt die Gefahr eines konsekutiven mechanischen Ileus. 


\subsubsection{Klinik und Diagnostik}

Klinisch bestehen Schmerzen, Fieber, Übelkeit und Erbrechen sowie nach einem kurzen schmerzfreien Intervall eine $\mathrm{Zu}$ nahme der Schmerzen und eine Abwehrspannung im rechten Oberbauch. Die freie Perforation mit einer galligen Peritonitis führt zu einem akuten Abdomen mit generalisierter Abwehrspannung. Die Zeichen eines mechanischen Ileus in der Röntgen-Abdomenübersicht mit Dünndarmspiegel, Aerobilie und Steinschatten im rechten Unterbauch weisen auf einen Gallensteinileus hin.

\subsubsection{Therapie}

Die Therapie der Gallenblasenperforation ist die notfallmäßige Cholezystektomie. In vielen Fällen ist die Konversion zur konventionellen Cholezystektomie erforderlich, sofern sie nicht schon primär intendiert ist. Der Gallensteinileus erfordert die Entfernung der obstruierenden Steine durch eine Enterotomie. Die Fistelsanierung und Cholezystektomie können in der gleichen Operation oder sekundär durchgeführt werden, falls der Patient dies in der initialen Operation nicht toleriert oder die pericholezystitische Entzündung so schwer ausgeprägt ist, dass die Cholezystektomie in der initialen Operation nicht sicher durchgeführt werden kann.

\subsection{Cholangiolithiasis}

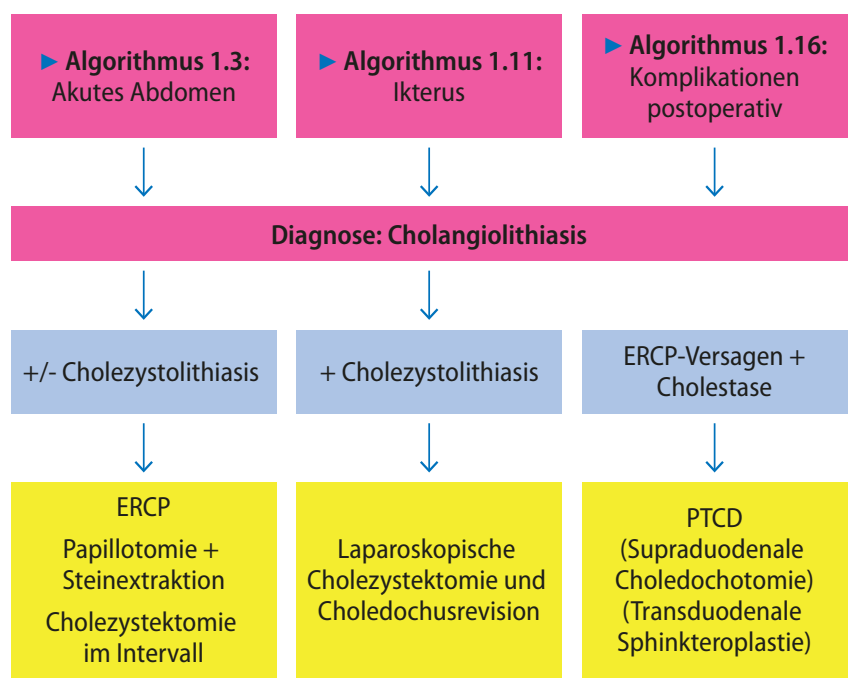

Die Cholangiolithiasis kann je nach Lokalisation in eine intrahepatische Lithiasis, Hepatikolithiasis, Choledocholithiasis, präpapilläre Lithiasis und Papillenkonkremente unterschieden werden (• Abb. 21.2). Die meisten Gallengangskonkremente sind sekundär und gelangen aus der Gallenblase über den Ductus cysticus in den DHC. Den primär in den Gallenwegen entstehenden Konkrementen liegt sehr häufig eine chronische Abflussbehinderung zugrunde (siehe Übersicht).

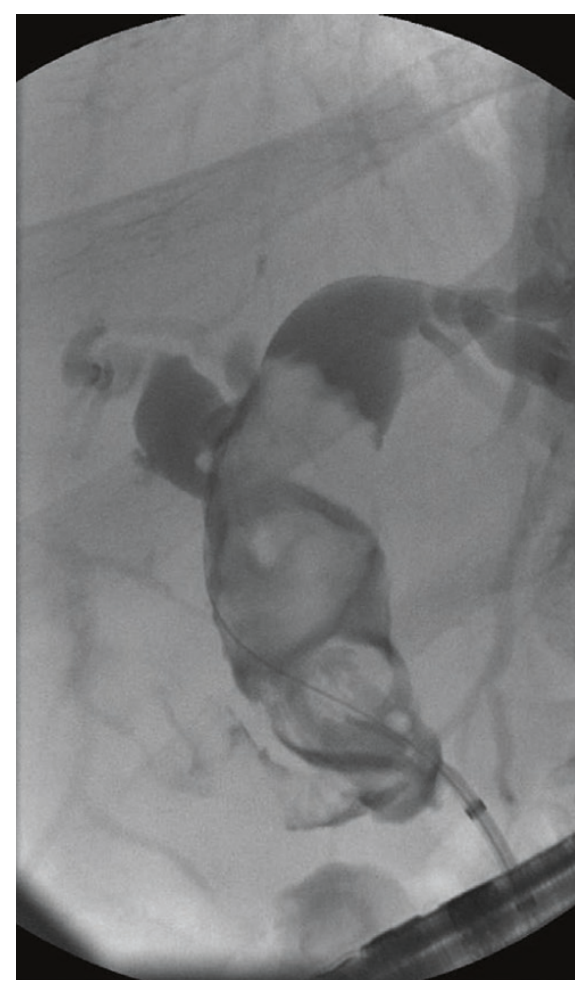

- Abb. 21.2 Cholangiolithiasis in beiden D. hepatici und DHC

Ursachen der primären Cholangiolithiasis

- Caroli-Syndrom

- Papillenstenose

- Pankreatitische DHC-Stenose

- Gallengangsstriktur

- Primär oder sekundär sklerosierende Cholangitis

- Langer Zystikusstumpf

- Nach Cholezystektomie

\subsubsection{Klinik und Diagnostik}

Anamnese, klinische Untersuchung, labormedizinische Untersuchungen (AP, $\gamma$-GT, Bilirubin, AST, ALT, Blutbild, CRP, Pankreasenzyme) und Oberbauchsonographie führen in aller Regel zur Diagnose. Leitsymptom ist wie bei der akuten Gallenblasenkolik der anfallsartige, an Intensität wechselnde kolikartige Schmerz im rechten Oberbauch mit Ausstrahlung in den Rücken und die rechte Schulter. Der akute Verschluss des DHC führt zum Auftreten einer Cholestase und eines schmerzhaften Ikterus (Verschlussikterus). Eine bakterielle Cholangitis und konsekutive intrahepatische cholangitische Abszesse als Folge der biliären Obstruktion können sich sekundär entwickeln und gehen mit Fieber und Schmerzen einher. In seltenen Fällen kann es zu einer Choledochusperforation mit Fistelbildung kommen. Eine chronische Cholestase führt zu rezidivierenden bakteriellen Cholangitiden und letztendlich zu einer sekundären biliären Zirrhose mit Zeichen der Leberatrophie. Eine sich langsam 
- Tab. 21.1 Differenzialdiagnose der Cholangiolithiasis

\begin{tabular}{|l|l|}
\hline & Differenzialdiagnose \\
$\begin{array}{l}\text { Benigne } \\
\text { Ursachen }\end{array}$ & $\begin{array}{l}\text { Mirizzi-Syndrom } \\
\text { Pankreatitis } \\
\text { Pankreaspseudozyste } \\
\text { Duodenalulkus } \\
\text { Sklerosierende Cholangitis } \\
\text { Primäre biliäre Zirrhose } \\
\text { Leberabszess } \\
\text { Papillenstenose } \\
\text { Juxtapapilläres Duodenaldivertikel } \\
\text { Choledochuszysten } \\
\text { Parasiten (Askariden, Lamblien) } \\
\text { HIV-assoziierte Cholangiopathie und Papillen- } \\
\text { stenose }\end{array}$ \\
\hline Maligne & $\begin{array}{l}\text { Gallengangskarzinom } \\
\text { Gallenblasenkarzinom } \\
\text { Pankreaskopfkarzinom } \\
\text { Papillenkarzinom } \\
\text { Hepatozelluläres Karzinom } \\
\text { Lebermetastasen }\end{array}$ \\
\hline
\end{tabular}

entwickelnde biliäre Obstruktion wird durch einen Pruritus und schmerzlosen Ikterus apparent und ist für das Vorliegen eines Tumors typisch. Ein transienter Steinabgang aus dem DHC über die Papille verursacht vorübergehende Schmerzen und eine Erhöhung der Cholestaseparameter. Eine Lipaseerhöhung weist auf eine Begleitpankreatitis hin. Durch die Sonographie ist der direkte Nachweis oder Ausschluss von Gallengangssteinen oft schwierig und gelingt häufig nicht. Eine Endosonographie oder Magnetresonanz-Cholangiographie (MR-C) kann in solchen Fällen die Diagnose sichern. Darüber hinaus ermöglicht eine endoskopische retrograde Cholangiographie (ERC) die Diagnosestellung und gleichzeitige Behandlung. Die Differenzialdiagnose der Cholangiolithiasis schließt stenosierende Prozesse der extrahepatischen Gallenwege, der Papille, des Pankreaskopfes, des Duodenums und zentrale Lebertumoren sowie parasitäre und infektiöse Erkrankungen ein (•Tab. 21.1).

\subsubsection{Therapie}

Symptomatische Gallengangssteine bedürfen wegen der drohenden Komplikationen wie Cholangitis, biliäre Pankreatitis und Sepsis einer zügigen Therapie. Die endoskopische Papillotomie mit Steinextraktion stellt das Standardverfahren dar.

Bei gleichzeitigem Vorliegen einer Cholezysto- und Choledocholithiasis wird bei entsprechender Expertise die laparoskopische Cholezystektomie und Choledochusrevision durchgeführt, ansonsten erfolgt ein zweizeitiges Vorgehen mit endoskopischer Papillotomie und Steinextraktion vor der Cholezystektomie, die möglichst zeitnah (innerhalb von Tagen bis zu 6 Wochen) erfolgen sollte.

Gelingt die endoskopische transpapilläre Therapie nicht, kann eine radiologische Intervention über einen perkutanen transhepatischen Cholangiodrainagenzugang (PTCD) erfolgen. Eine chirurgische Exploration des DHC durch eine supraduodenale Choledochotomie sollte als letzte Alternative angesehen werden: Nach der Cholezystektomie erfolgt die Inzision des Retroperitoneums zur Mobilisation des Duodenums nach Kocher. Der DHC wird knapp oberhalb des Duodenums 1-2 cm längs eröffnet. Nach Anlage zweier Haltefäden wird ein Fogarty-Katheter in den DHC eingeführt und durch Palpation die Passage des Katheters in das Duodenum kontrolliert. Der Ballon des Fogarty-Katheters wird anschließend mit einer Spritze aufgefüllt, gegen die Papille zurückgezogen, dann vorsichtig soweit entleert und behutsam zurückgezogen, bis er die Papille passieren kann. Danach wird der Ballon wieder aufgefüllt und behutsam mit den Konkrementen bis zur Choledochotomie zurückgezogen. Diese können dann entfernt werden. Anschließend sollte sich der Operateur vergewissern, dass das Gallengangsystem keine weiteren Pathologien aufweist. Die intraoperative Cholangiographie nach Einlage einer T-Drainage über die Choledochotomie und Verschluss derselben ist eine Möglichkeit, das Gallengangsystem zu kontrollieren. Die T-Drainage ermöglicht es weiterhin, die durch die Manipulationen spastische und ödematös geschwollene Papille zu entlasten. Die transduodenale Sphinkteroplastie ist im Falle eines impaktierten Konkrements in der Ampullenregion indiziert. Sie beinhaltet die Inzision der Papille mit partieller Naht der Schnittränder, ohne den Sphinkter des DHC oder des Ductus pancreaticus einzubeziehen. Es erfolgt die Inzision des Retroperitoneums zur Mobilisation des Duodenums nach Kocher, weil eine gute Exposition und Mobilisation des Duodenums die Identifikation der Papille erleichtert und einen mühelosen Verschluss des Duodenums erlaubt.

\subsection{Verschlussikterus}

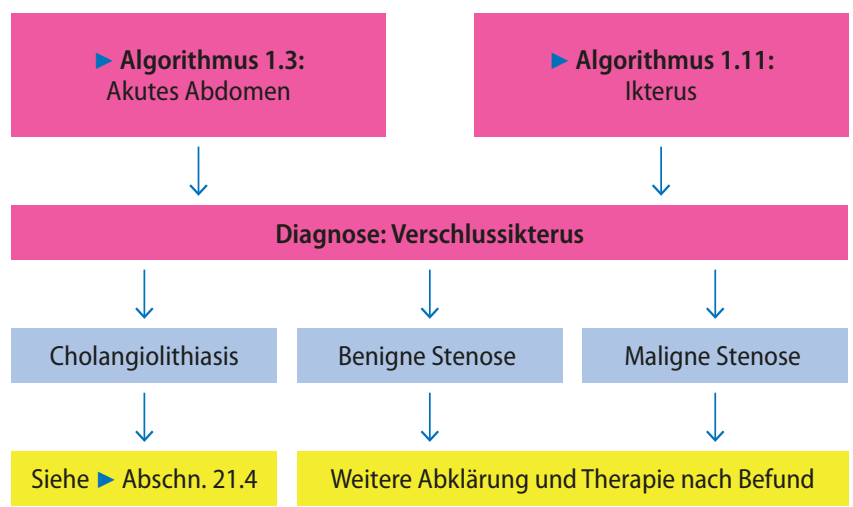

Die mechanische Obstruktion der Gallenwege wird als Verschlussikterus bezeichnet. Die Choledocholithiasis ist die häufigste Ursache eines Verschlussikterus. Karzinome und entzündliche Veränderungen des hepato-bilio-pankreatischen Systems können ebenfalls zu einem Verschlussikterus führen. Weitere Ursachen sind Parasiten (Askariden, Echino- 
kokken) und die Kompression des Gallengangs von außen durch Tumoren oder durch ein Mirizzi-Syndrom.

\subsubsection{Klinik und Diagnostik}

Leitsymptome sind der Ikterus von Haut und Skleren, die Dunkelfärbung des Urins und die Entfärbung des Stuhls. Die Anamnese kann wegweisend für die Ursache sein. Ein Verschlussikterus mit kolikartigen Schmerzen weist auf eine Choledocholithiasis hin, während ein schmerzloser Verschlussikterus bis zum Beweis des Gegenteils für ein Malignom spricht. In den labormedizinischen Untersuchungen sind die Cholestaseparameter AP, $\gamma$-GT und Bilirubin erhöht. Ein Anstieg der Transaminasen deutet auf eine Cholangitis und der Nachweis von erhöhter Amylase und Lipase auf eine Begleitpankreatitis hin. Bei langandauernder Cholestase kommt es zu einem Anstieg des INR-Werts bzw. Erniedrigung der Prothrombinzeit (Quick-Wert). Die Sonographie mit Nachweis einer intra- und extrahepatischen Cholestase und Erweiterung des DHC ist die primäre Diagnostik. Es gelingt jedoch nicht immer, den DHC bis zur Papille darzustellen. Eine Endosonographie, MR-C oder CT kann bei unklaren Befunden erfolgen.

\subsubsection{Therapie}

Eine schnelle Abklärung und Therapie der Gallenwegsobstruktion ist aufgrund der möglichen Komplikationen unumgänglich. Die Therapie richtet sich nach der Ursache des Verschlussikterus. Eine Choledocholithiasis sollte innerhalb von 24-48 Stunden behandelt werden ( $\triangleright$ Abschn. 21.4). Die konventionelle Cholezystektomie mit Gallengangsrevision und ggf. Rekonstruktion der Gallenwege ist die Therapie der Wahl beim Mirizzi-Syndrom. In spezialisierten Zentren wird beim Mirizzi-Syndrom bei entsprechender Expertise die laparoskopische Cholezystektomie und Choledochusrevision durchgeführt. Malignome erfordern in Abhängigkeit von der Lokalisation neben der Gallengangsresektion auch eine Leberresektion oder eine pyloruserhaltende partielle Pankreatoduodenektomie bzw. eine Whipple-Operation.

\subsection{Cholangitis}

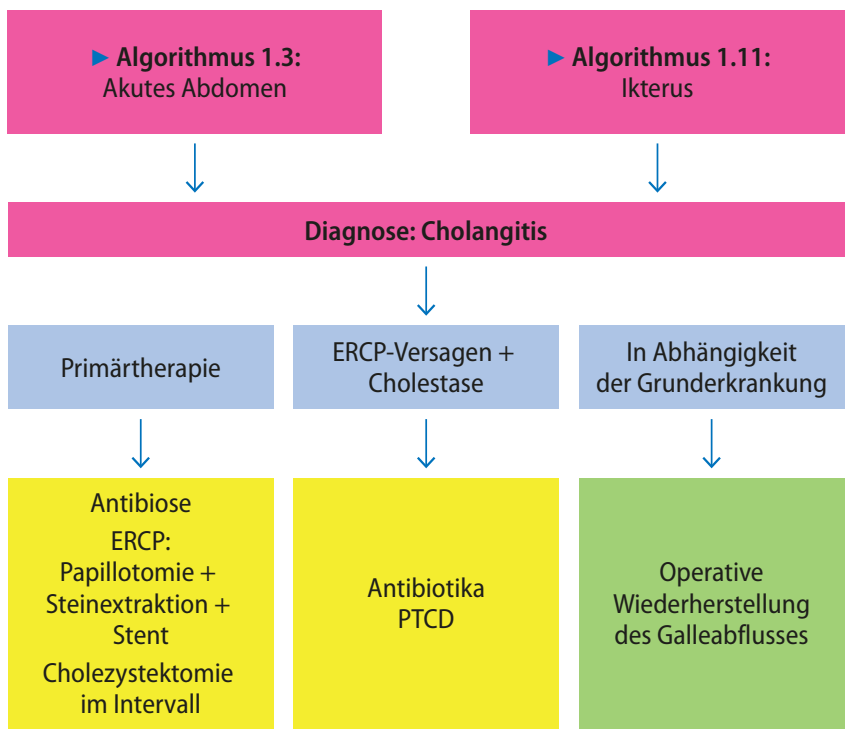

Die Cholangitis ist eine Infektion der Gallenwege, die mit einer biliären Obstruktion, einer damit verbundenen Abflussbehinderung der Gallenflüssigkeit sowie einer Kolonisation der Gallenflüssigkeit mit Bakterien oder Pilzen assoziiert ist. Es kann jede Lokalisation des Gallenwegsystems betroffen sein. Die akute Cholangitis zeigt mitunter schwere Verläufe bis hin zu einer Sepsis mit Multiorganversagen. Die rezidivierenden Entzündungen führen zu einer chronischen Cholangitis, die bei längerem Bestehen eine sekundäre biliäre Zirrhose zur Folge haben kann. Die häufigsten Ursachen für eine biliäre Obstruktion sind Gallenwegskonkremente, benigne oder maligne Strikturen/Stenosen, kongenitale Anomalien, sklerosierende Cholangitiden, stenosierte Gallengangsstents oder -drainagen und eine Infektion durch Parasiten (-Tab. 21.2). Im Verlauf nach Operationen (Anlage einer biliodigestiven Anastomose) oder interventionellen Manipulationen (endoskopische retrograde Cholangio-Pankreatikographie - ERCP; perkutane transhepatische Cholangiodrainage - PTCD) an den Gallenwegen kann es zu einer Cholangitis kommen, die sich kanalikulär, hämatogen und lymphogen ausbreiten kann. Die häufigsten Erreger sind gramnegative Koli-Bakterien, Mischinfektionen mit Enterokokken, Streptokokken, Proteus, Klebsiellen und Candida.

\subsubsection{Klinik und Diagnostik}

Die Charcot-Trias beschreibt die Leitsymptome einer akuten Cholangitis: kolikartige Schmerzen im rechten Oberbauch, intermittierendes Fieber mit Schüttelfrost und Ikterus. Die Anamnese, klinische Untersuchung (Hepatomegalie) und labormedizinische Erhöhung der Entzündungsparameter (Blutsenkungsgeschwindigkeit [BSG], Leukozyten und CRP) weisen auf eine Infektion hin. Weiterhin sind das Bilirubin und die Cholestaseparameter (AP, $\gamma$-GT) erhöht. Die Trans- 
- Tab. 21.2 Ursachen einer Cholangitis

\begin{tabular}{l|l}
$\begin{array}{l}\text { Gallengangs- } \\
\text { steine }\end{array}$ & $\begin{array}{l}\text { Choledocholithiasis } \\
\text { Hepatolithiasis } \\
\text { Mirizzi-Syndrom }\end{array}$ \\
$\begin{array}{l}\text { Benigne } \\
\text { Striktur/ }\end{array}$ & $\begin{array}{l}\text { Postoperative Komplikationen nach Gallen- } \\
\text { wegschirurgie } \\
\text { Chronische Pankreatitis } \\
\text { Primäre sklerosierende Cholangitis } \\
\text { Orthotope Lebertransplantation } \\
\text { HIV-Cholangiopathie }\end{array}$ \\
\hline Malignome & $\begin{array}{l}\text { Intrahepatisches Cholangiokarzinom } \\
\text { Gallenblasenkarzinom } \\
\text { Hiläres Cholangiokarzinom (perihiläres } \\
\text { Karzinom) } \\
\text { Distales Gallengangskarzinom } \\
\text { Ampullenkarzinom } \\
\text { Papillenkarzinom } \\
\text { Pankreaskopfkarzinom } \\
\text { Duodenalkarzinom }\end{array}$ \\
\hline $\begin{array}{l}\text { Interventionen } \\
\text { an den Gallen- } \\
\text { wege }\end{array}$ & $\begin{array}{l}\text { Endoskopische retrograde Cholangio- } \\
\text { Pankreatikographie (ERCP) } \\
\text { Perkutane transhepatische Cholangio- } \\
\text { drainage (PTCD) } \\
\text { Gallengangsstent }\end{array}$ \\
\hline Parasiten & $\begin{array}{l}\text { Askariden } \\
\text { Echinokokkose } \\
\text { Lamblien }\end{array}$ \\
\hline
\end{tabular}

aminasen (ALT, AST) können bei einer Mitbeteiligung bzw. Schädigung des Leberparenchyms erhöht sein. Die Oberbauchsonographie weist in den meisten Fällen eine Erweiterung der intra- und extrahepatischen Gallengänge nach. Eine MR-C ist dann indiziert, wenn der sonographische Befund unklar bleibt. Bei einem initialen Malignomverdacht wird eine CT- oder MRT-Untersuchung durchgeführt.

\subsubsection{Therapie}

Die initiale Therapie der akuten Cholangitis sollte neben der hämodynamischen Stabilisierung des Patienten folgende Prinzipien verfolgen: parenterale Breitspektrumantibiotika und rechtzeitige Entlastung der biliären Obstruktion als Grundstein der Behandlung einer akuten Cholangitis. Die bevorzugte Methode der Entlastung der biliären Obstruktion ist abhängig von der Verfügbarkeit und Expertise vor Ort, der Ätiologie, der Lokalisation und Schwere der Obstruktion sowie dem klinischen Zustand des Patienten.

Patienten, welche auf eine initiale parenterale AntibiotikaBehandlung nicht innerhalb der ersten 12-24 Stunden ansprechen oder deren Zustand sich rapide verschlechtert, müssen einer notfallmäßigen nicht operativen Entlastung der Gallenwege zugeführt werden. Im Falle einer distal der Bifurkation gelegenen Obstruktion ist die bevorzugte Methode eine endoskopische Entlastung durch eine ERC mit Papillotomie und transpapillärer Stentimplantation in den DHC zur Dekompression des Gallenwegsystems. Die Papillotomie ist bei einer bestehenden Koagulopathie, Cholangiosepsis und hämodynamischer Instabilität kontraindiziert. Die Entlastung durch eine PTCD sollte dann durchgeführt werden, wenn wegen frustraner Intubation der Papille und des DHC eine ERC nicht durchgeführt werden kann. Sie ist insbesondere beim Vorliegen hilärer oder intrahepatischer Obstruktionen eine Alternative. In den Fällen, in denen eine definitive Beseitigung der biliären Obstruktion als kurative Therapie zur Wiederherstellung des Gallenabflusses und der damit verbundenen Rezidivprophylaxe einer Cholangitis nur durch eine Operation erfolgen kann, sollte diese nach Stabilisierung des Patienten, Abklingen des Ikterus (Bilirubin $<5 \mathrm{mg} / \mathrm{dl}$ ) und im infektionsfreien Intervall erfolgen. Die hierbei zu wählenden Operationsverfahren richten sich nach den Ursachen und Lokalisationen der biliären Obstruktion und umfassen das ganze Spektrum der hepatobiliären und Pankreaschirurgie.

\subsection{Benigne Gallengangsstrikturen}

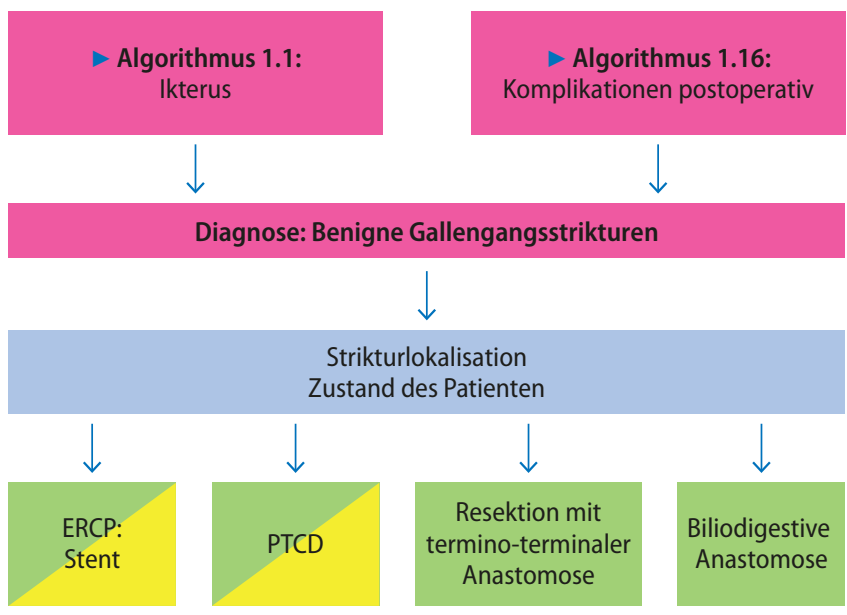

Die häufigsten Gallengangsstrikturen resultieren aus iatrogenen Gallengangsverletzungen im Rahmen einer Cholezystektomie (-Abb. 21.3). Die meisten Verletzungen entstehen entweder durch technische Fehler oder durch eine Fehleinschätzung der anatomischen Verhältnisse. Insbesondere können bei einer akuten Cholezystitis schwere entzündliche Veränderungen im Calot-Dreieck die Identifikation der anatomischen Strukturen erschweren oder unmöglich machen.

Ca. $10 \%$ der Gallengangsstrikturen sind Folgen einer Gallengangsrevision, Gallengangsanastomose oder biliodigestiven Anastomose. Andere Oberbauchoperationen an Leber, Pankreas, Magen und Duodenum können ebenfalls das Gallenwegssystem gefährden, gleichwohl die Inzidenz der Gallenwegsverletzung bei diesen Operationen sehr gering ist. Weitere Ursachen sind u. a. die Choledocholithiasis, die kongenitale Gallengangsatresie, die chronisch rezidivierende Cholangitis, die sklerosierende Cholangitis, die chronische Pankreatitis, das chronische Duodenalulkus und Traumata. 


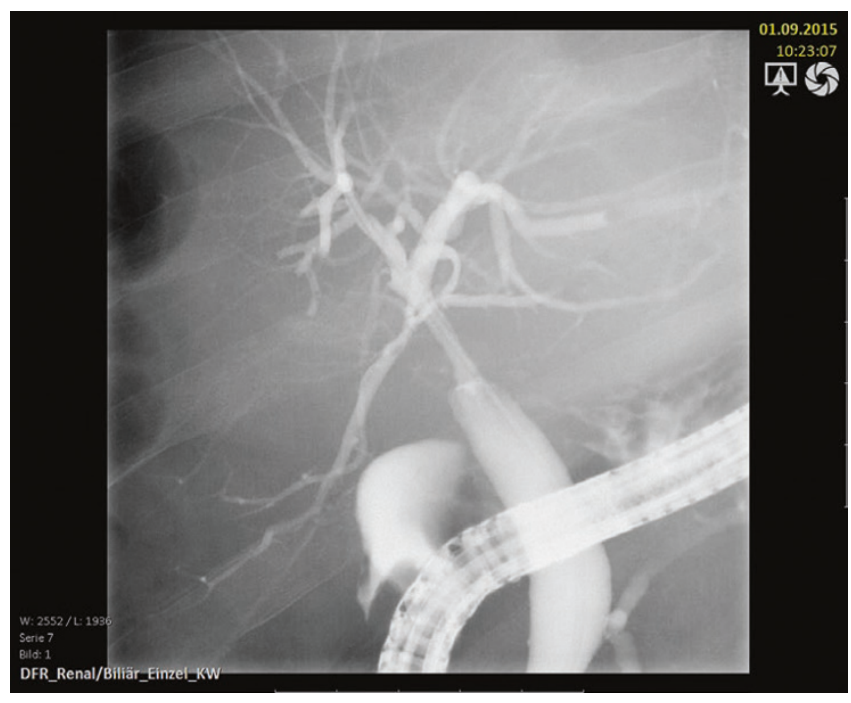

- Abb. 21.3 Gallengangsstriktur nach laparoskopischer Cholezystektomie

Ein pathogenetischer Faktor der Gallengangsstrikturen ist die fibrosierende Wirkung der Gallenflüssigkeit auf das umliegende Gewebe nach dem Austritt bei einer Gallengangsverletzung. Es resultiert eine ausgeprägte Narbenbildung, welche die Entwicklung von Strikturen oder Stenosen induziert. Ein weiterer Faktor könnte eine durch ausgedehnte intraoperative periduktale Dissektion induzierte Ischämie des DHC sein. Hierbei wird die axiale Blutversorgung des extrahepatischen Gallengangssystems beeinträchtigt, was zu ischämischen Gallengangsstrikturen führt.

\subsubsection{Klinik und Diagnostik}

Die Anamnese mit vorausgegangenen Operationen an den Gallenwegen ist wegweisend. In Abhängigkeit vom Grad der Stenosierung können Oberbauchschmerzen, Ikterus und Cholangitis auftreten. In den labormedizinischen Untersuchungen findet sich eine Erhöhung der Cholestaseparameter $\gamma$-GT, AP und Bilirubin. Im Falle einer segmentalen Obstruktion kann auch ein normales Bilirubin vorliegen. Die Diagnose erfolgt primär durch eine Sonographie mit Nachweis erweiterter Gallengänge. Die weiterführende Diagnostik erfolgt durch eine ERCP. Bei Vorliegen einer biliodigestiven Anastomose ist häufig eine MR-C erforderlich. Die präoperative Diagnostik schließt insbesondere dann eine CT- oder MRT-Untersuchung ein, wenn aufgrund einer segmentalen Cholestase mit konsekutiver biliärer Zirrhose und sekundärer Leberatrophie eine Leberteilresektion erforderlich ist.

\subsubsection{Therapie}

Die Therapie von Gallengangsstrikturen richtet sich nach der Lokalisation der Enge. Strikturen nach Cholezystektomien im proximalen oder mittleren Drittel des DHC können endoskopisch durch eine Stenteinlage dilatiert werden. Gelingt dies

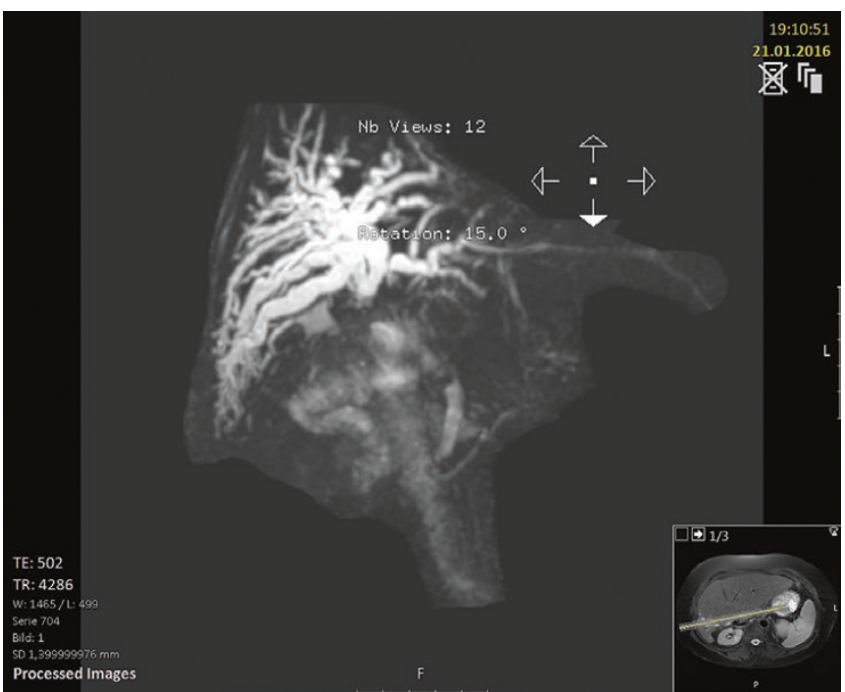

- Abb. 21.4 Biliäre Gallengangsstriktur des rechten Gallengangsystems

nicht, sollte eine kurzstreckige Stenose reseziert und der DHC nach Möglichkeit termino-terminal anastomosiert werden. Bei langstreckigen Stenosen des DHC und Stenosen im Bereich der Hepatikusgabel ist in aller Regel die Anlage einer biliodigestiven Anastomose die Therapie der Wahl. Bei Patienten, bei denen eine Operation aufgrund bestehender Kontraindikationen, z. B. schwere Sepsis aufgrund einer akuten Cholangitis, nicht durchgeführt werden kann, sollte alternativ eine PTCD angelegt werden. Liegt eine segmentale Cholestase aufgrund einer langanhaltenden Obstruktion mit chronischer Cholangitis, konsekutiver biliärer Zirrhose und sekundärer Leberatrophie der betreffenden Segmente vor, ist eine Leberteilresektion, ggf. mit Anlage einer biliodigestiven Anastomose, erforderlich (• Abb. 21.4).

\subsection{Portale Hypertension}

Eine portale Hypertension liegt dann vor, wenn eine Behinderung im Abstromgebiet der Pfortader zu einer Erhöhung des Druckgradienten zwischen Pfortader und V. cava inferior von mehr als $6 \mathrm{mmHg}$ führt. Die Ursachen der portalen Hypertension sind in - Tab. 21.3 dargestellt. Die Folge der portalen Hypertension ist eine Ektasie des vorgeschalteten Venensystems mit Entwicklung einer Varikosis im Magen und Ösophagus, die zu lebensbedrohlichen Blutungen führen kann.

\subsubsection{Klinik und Diagnostik}

Die Diagnose der portalen Hypertension und seiner Ursache erfolgt anhand der klinischen Präsentation, Anamnese und Untersuchung sowie weiterführender Untersuchungen mittels Labor mit Serologie, diagnostischer Aszitespunktion, Sonographie, Duplexsonographie, Endoskopie, Leberpunktion, CT und MRT. 
- Tab. 21.3 Ursachen der portalen Hypertension

\begin{tabular}{|l|l|}
\hline Präsinusoidal & Extrahepatisch: \\
& - Pfortaderthrombose \\
& - Milzvenenthrombose \\
& - Arterioportaler Shunt \\
& Intrahepatisch: \\
& - Bilharziose (Schistosomiasis) \\
& - Kongenitale Leberfibrose \\
& - Venookklusive Erkrankung (VOD) \\
& - Medikamente und Toxine \\
\hline Sinusoidal & Zirrhose \\
\hline Postsinusoidal & Budd-Chiari-Syndrom \\
& VOD \\
\hline & Kongestive Herzinsuffizienz \\
\hline
\end{tabular}

$\checkmark$ In dem überwiegenden Anteil der Fälle ist eine Leberzirrhose die Ursache der portalen Hypertension.

In der Anamnese ist nach chronischer Lebererkrankung, Ikterus, Hepatitis, Infektionen, Thrombosen, Herzinsuffizienz und Medikamenten zu fragen. Bei der klinischen Untersuchung ist auf Leber- und Milzgröße, Spider naevi, Caput medusae, Aszites mit Bauchumfangsvermehrung, Gewichtszunahme, Dyspnoe durch Zwerchfellhochstand und Pleuraerguss, Beinödeme sowie hepatische Enzephalopathie zu achten.

Die akute obere gastrointestinale Blutung aus Ösophagusund Korpus-/Fundusvarizen mit Aszites zeigt das Endstadium einer Leberzirrhose an. Es liegen ggf. weitere Komplikationen wie eine Splenomegalie, Hyperspleniesyndrom, spontan bakterielle Peritonitis und hepatorenales Syndrom vor.

\subsubsection{Therapie}

Siehe dazu auch • Abb. 21.5, - Abb. 21.6 und • Abb. 21.7.

Die akute Varizenblutung erfordert neben der sofortigen Notfallendoskopie mit dem Ziel der Diagnose, Lokalisierung und Blutstillung eine medikamentöse Senkung des portalen Drucks. In ca. 90 \% der Fälle kann die akute Varizenblutung mittels endoskopischer und medikamentöser Behandlung erfolgreich therapiert werden. Falls eine endoskopische Blutstillung nicht zu erreichen ist oder diese mehrfach rezidiviert, ist die interventionelle Anlage eines transjugulären intrahepatischen portosystemischen Shunts (TIPS) indiziert. Die Notfalloperationen in Form von Ösophagustranssektion, Magenwandsperroperation und Anlage eines portosystemischen Shunts bedürfen wegen der hohen Mortalität von $>50 \%$ der genauen Indikationsstellung und kommen nur in den seltenen Fällen in Betracht, in denen die endoskopische Blutstillung oder die Anlage eines TIPS nicht möglich ist.

Die Primärprophylaxe einer Varizenblutung ist bei erhöhtem Blutungsrisiko - Vorliegen einer Leberzirrhose im Child-Pugh-Stadium C und Varizen größer $5 \mathrm{~mm}$ sowie Varizen mit red colour sign - indiziert und wird medikamentös mit nicht selektiven Betablockern (z. B. Propranolol und Carvedilol) und endoskopischer Ligatur vorgenommen.

Die Sekundärprophylaxe nach der 1. Blutung erfolgt ebenfalls mit nicht selektiven Betablockern und durch Ligaturbehandlungen. Im Falle der Rezidivblutung trotz Sekundärprophylaxe können portosystemische Shuntverfahren mit dem Ziel der Senkung des portokavalen Druckgradienten durchgeführt werden. Hierzu gehören der TIPS und die chirurgischen portosystemischen Shunts (•Tab. 21.4, - Abb. 21.8). Eine eingeschränkte Leberfunktion und eine therapierefraktäre Enzephalopathie stellen Kontraindikationen dar, sodass in diesen Fällen ggf. eine Lebertransplantation indiziert ist.

Es wird für die Behandlung des Aszites, der spontanen bakteriellen Peritonitis (SBP) und des hepatorenales Syndroms auf die S3-Leitlinie verwiesen. Ein TIPS kann in ca. $70 \%$ den Aszites vermindern oder beseitigen und im Falle eines therapierefraktären Aszites eine gute Alternative im Vergleich zu rekurrenten Parazentesen sein.

Der TIPS stellt für Patienten im Child-Pugh-Stadium C eine Kontraindikation dar. Diese Patienten sollten durch eine Lebertransplantation behandelt werden.

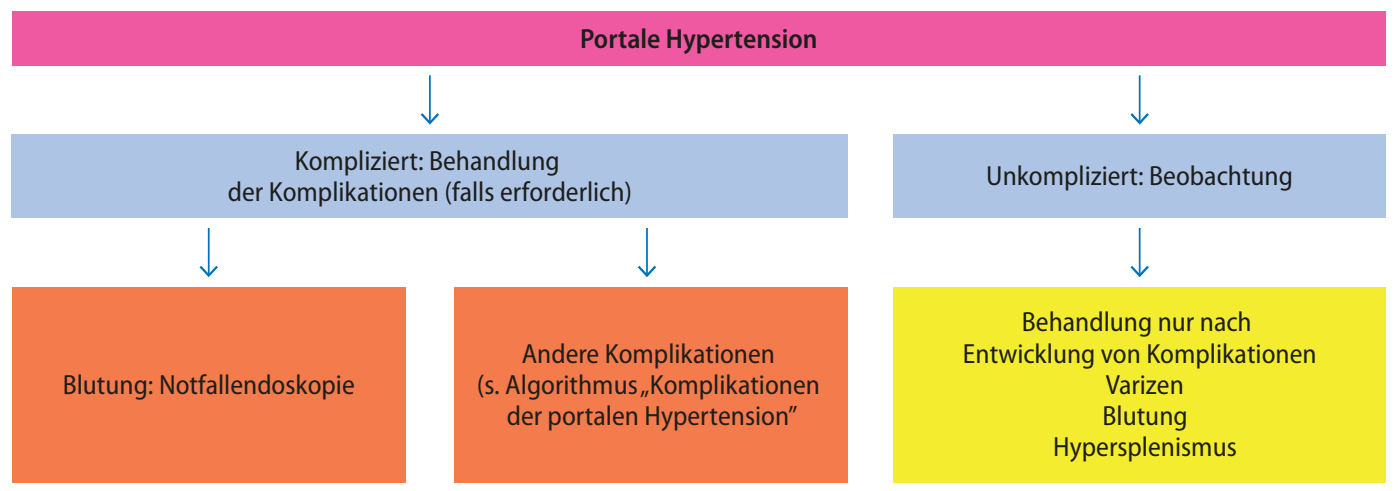

- Abb. 21.5 Algorithmus bei portaler Hypertonie 


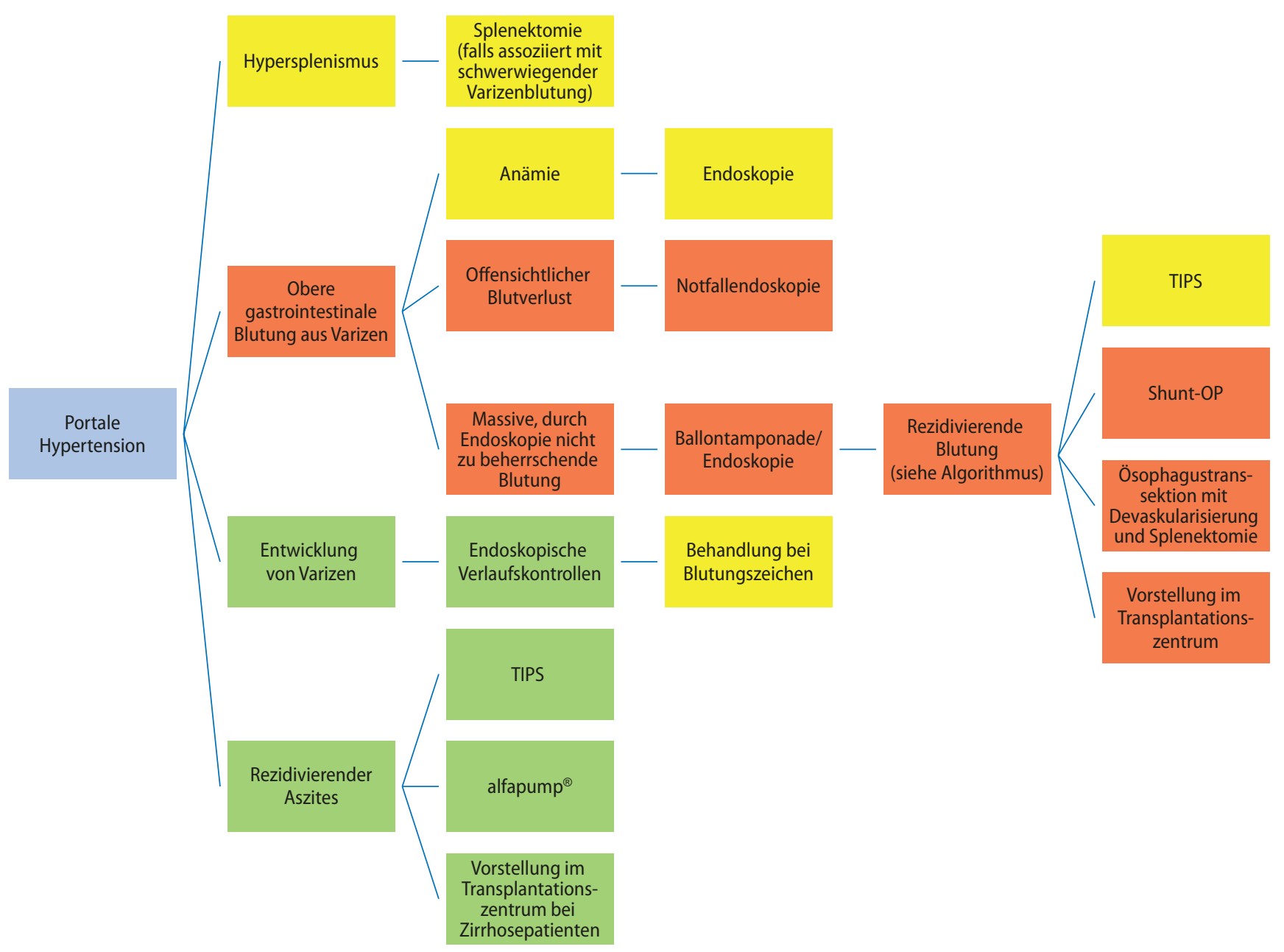

- Abb. 21.6 Algorithmus bei Komplikationen der portalen Hypertonie

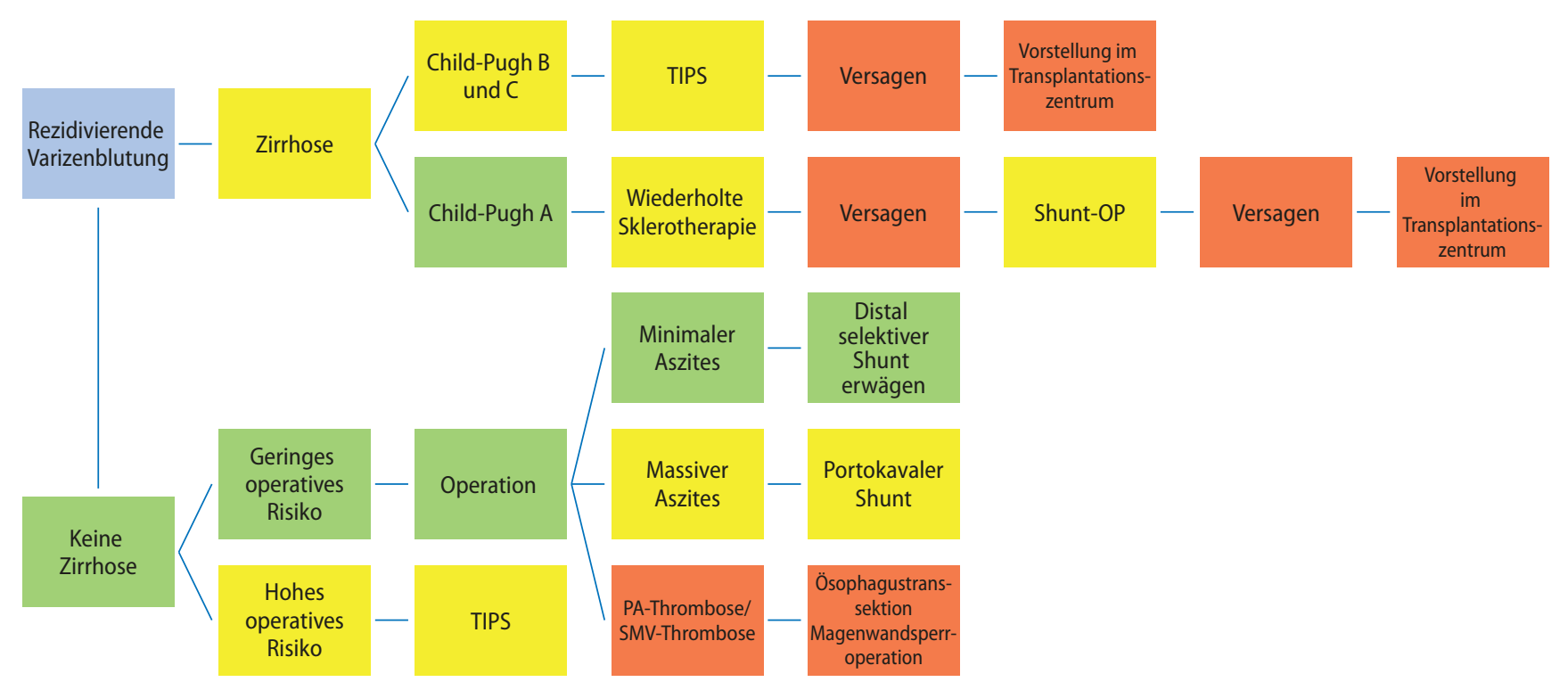

- Abb. 21.7 Algorithmus bei rezidivierenden Varizenblutung 

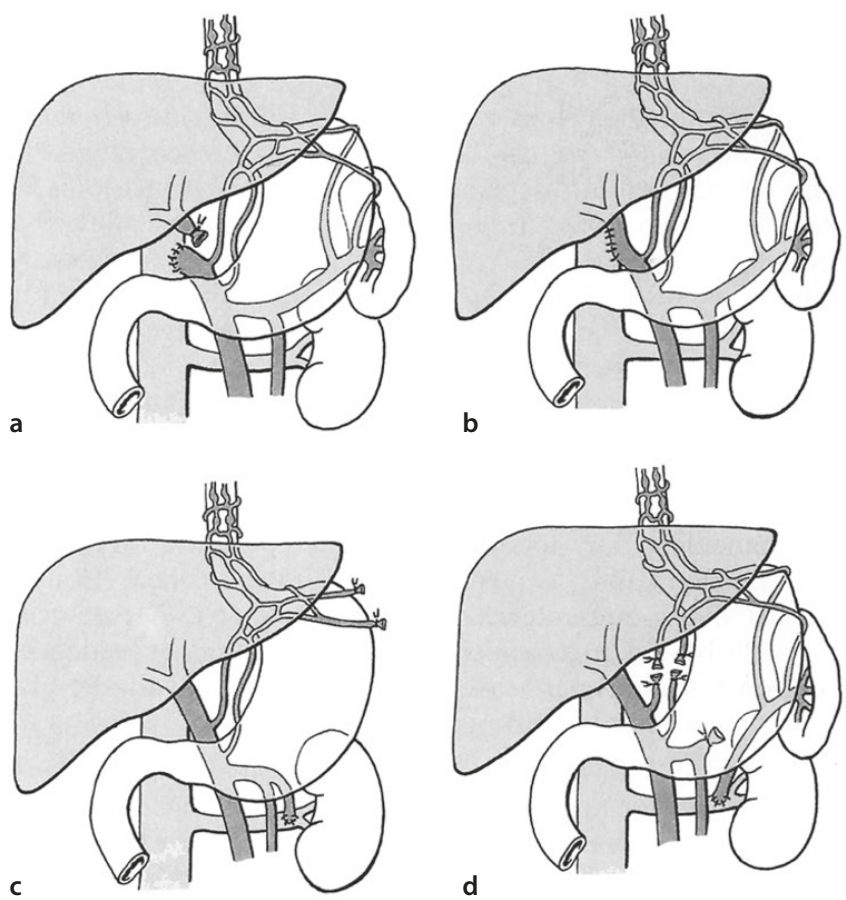

d
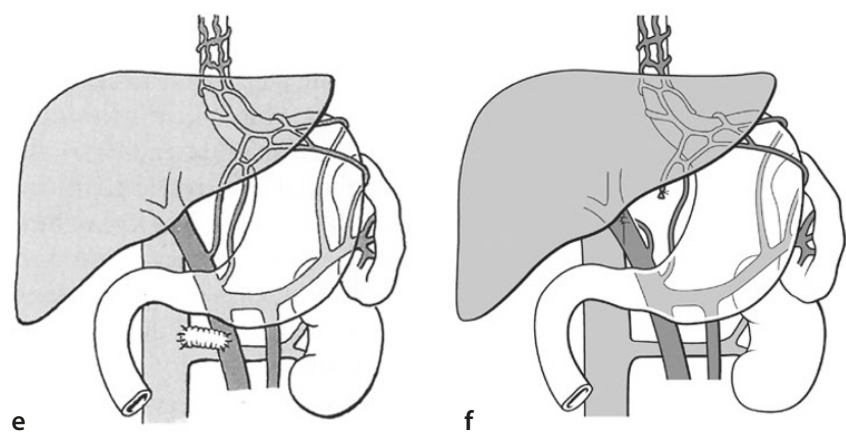

- Abb. 21.8 Shuntformen. a Portokavaler End-zu-Seit-Shunt, b portokavaler Seit-zu-Seit-Shunt, c proximaler splenorenaler Endzu-Seit-Shunt, d distaler splenorenaler End-zu-Seit-Shunt (Warren), e mesenterikokavaler Seit-zu-Seit-Shunt mit Interponat (nach Drapanas 1972), f Inokuchi-Shunt der V. gastrica sinistra auf die V. cava inferior (Aus Nagel u. Löhlein 2006)

- Tab. 21.4 Chirurgische portosystemische Shunts

Komplette Portokaval end-zu-seit oder seit-zu-seit Shunts Mesentericokavaler H-Shunt nach Drapanas Proximal splenorenal nach Linton Laterolateral splenorenal nach Cooley

Selektiver Distal splenorenal nach Warren

Shunt V. coronaria ventriculi auf V. caca inferior nach Inokuchi

\subsection{Pyogener Leberabszess}

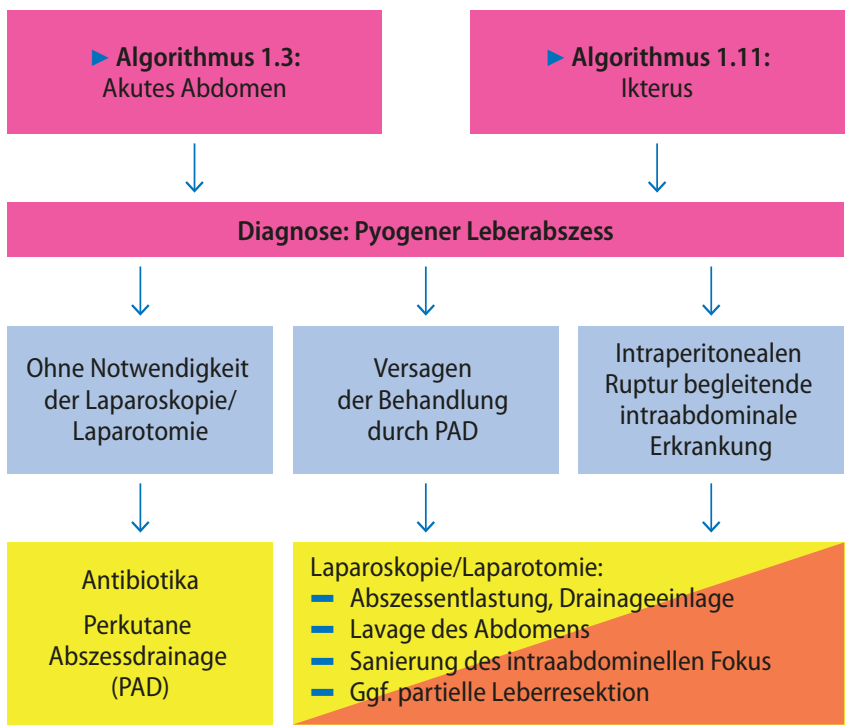

Der pyogene Leberabszess ist eine seltene, aber lebensbedrohliche Erkrankung mit hoher Morbidität und Mortalität. Er ist definiert als eine solitäre oder multiple Ansammlung von Eiter innerhalb der Leber.

Leberabszesse als Folge von Erkrankungen der Gallenwege, insbesondere solche, die zu Gallengangsstrikturen oder -stenosen führen, stellen die häufigste Ursache dar. Die primäre und sekundäre Cholangitis sowie angeborene Gallenwegsanomalien können zu einer Cholangiosepsis mit Bildung von Leberabszessen führen. Die zunehmend aggressive interventionelle und chirurgische Behandlung hepatobilärer Erkrankungen durch Gallengangsstents, perkutane transhepatische Gallengangsdrainagen und ausgedehnte Leberresektionen stellen weitere Ursachen für die Entstehung von Leberabszessen dar. Ferner kann es zu einer hämatogenen Ausbreitung gastrointestinaler oder pelviner Infektionen über das Pfortaderstromgebiet und die Leberarterie kommen. Es können daraus septische Verschleppungen und Abszedierung in der Leber resultieren. Eine neonatale Nabelschnursepsis kann sich ebenfalls in die Pfortader ausbreiten und nachfolgend zu einem Leberabszess führen.

Schädigungen des hepatischen arteriellen Systems - z. B. verursacht im Rahmen einer Cholezystektomie oder einer Thrombose der A. hepatica nach Lebertransplantation - können zu einem Leberabszess führen. Diese können auch nach interventionellen Behandlungen von hepatischen Malignomen durch transarterielle Chemoembolisation (TACE), selektive intrahepatische Radiotherapie (SIRT) und lokale perkutane Ablationen wie Radiofrequenzablation (RFA) oder Mikrowellenablation (MWA) entstehen - Abb. 21.9).

Die häufigsten relevanten Erreger sind Escherichia coli, Klebsiella spp., Enterococcus spp. Bacteroides spp. und Fusobacterium spp. 


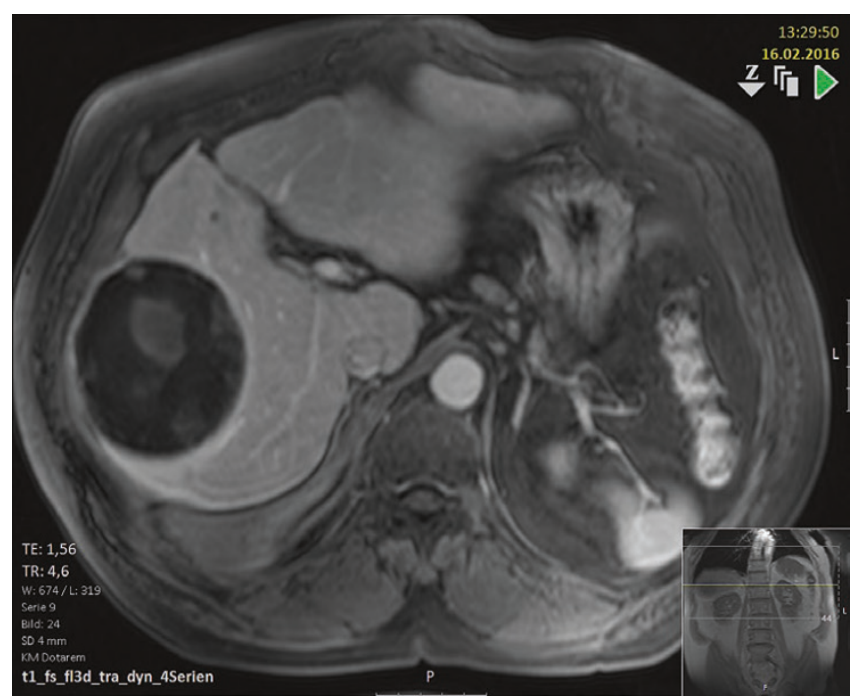

- Abb. 21.9 Abszess nach interventioneller Mikrowellenablation eines Rezidivs bei hepatozellulärem Karzinom (HCC)

\subsubsection{Klinik und Diagnostik}

Die Patienten stellen sich mit einem allgemeinen Krankheitsgefühl und unspezifischen Symptomen vor. Die häufigsten klinischen Erscheinungen sind Fieber, Schüttelfrost und Druckschmerz im rechten Oberbauch. Ein breites Spektrum unspezifischer Symptome wie Übelkeit, Erbrechen, Durchfall, Gewichtsverlust, Ikterus, Myalgien und Kopfschmerzen können ebenfalls auftreten. Eine Dyspnoe kann Hinweis für einen konsekutiven, rechtsseitigen Pleuraerguss oder einen Zwerchfellhochstand sein.

Die häufigsten labormedizinischen Veränderungen sind der Nachweis einer Anämie, Leukozytose, eines erhöhtes CRP, einer Erhöhung der Alanin-Aminotransferase (ALAT) und der alkalischen Phosphatase (AP). Der Ultraschall und die Computertomographie ermöglichen die Lokalisation des Abszesses und eine Entnahme einer Probe für die mikrobiologische Abklärung durch eine gesteuerte Punktion und gleichzeitige Anlage einer perkutanen Abszessdrainage (PAD). Das CT ermöglicht eine komplette Untersuchung des Abdomens und des Beckens, um eine ursächliche Erkrankung zu detektieren, welche einer weiterführenden Therapie bedarf. Im Falle von Auffälligkeiten der intra- und extrahepatischen Gallenwege in der CT-Untersuchung, insbesondere bei Verdacht auf ein hepatobiliäres Malignom, kann eine Magnetresonanztomographie/Magnetresonanz-Cholangiopankreatikographie (MRT/MRCP) sinnvoll sein.

\subsubsection{Therapie}

Die Basis der Therapie des Leberabszesses liegt in der kompletten Ableitung des Pus und des infizierten Debris, der Einleitung einer adäquaten Antibiotikatherapie und der Behebung der zugrunde liegenden Ursache. Der Ausschluss eines Amöbenabszesses als mögliche Differenzialdiagnose ist unverzichtbar. Dieser wird im Gegensatz zum pyogenen Abszess gemeinhin wegen des sehr guten Ansprechens mit Antibiotika behandelt.

Eine antibiotische Therapie sollte nach Diagnosestellung und Entnahme einer mikrobiologischen Probe aus dem Abszess oder Blutkulturen mit einem Breitbandspektrumantibiotikum begonnen werden. Die initiale Antibiotikatherapie erfolgt entweder mit einem Cephalosporin der 3. Generation und Metronidazol oder Piperacillin/Tazobactam und sollte im Verlauf dem Antibiogramm der mikrobiologischen Untersuchungen angepasst werden. Die Dauer der Behandlung richtet sich nach dem Ansprechen der Therapie. Dieses wird durch Ultraschallkontrollen des Leberabszesses, Rückgang des Fiebers und der labormedizinischen Entzündungsparameter sowie Besserung der klinischen Symptome dokumentiert.

Die interventionelle Therapie mittels PAD ist das Therapieverfahren der Wahl. Damit können sowohl solitäre als auch multiple Abszesse sicher und effektiv behandelt werden.

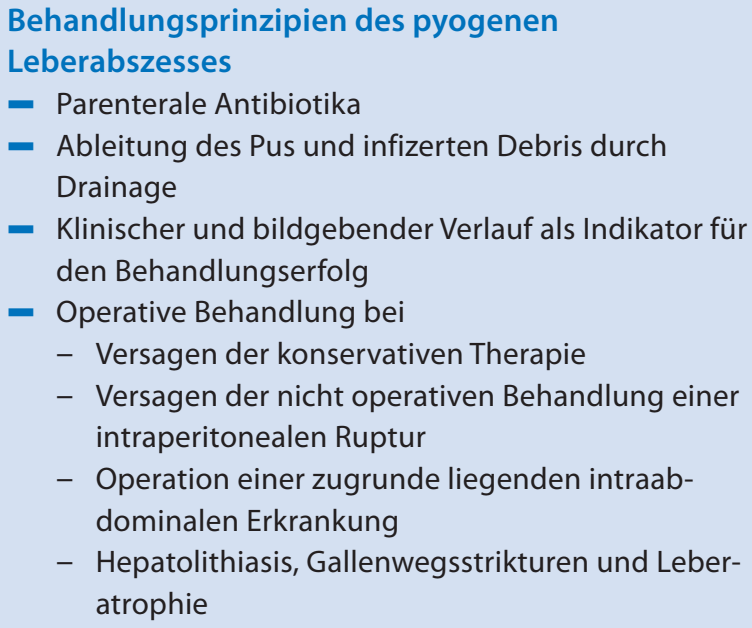

Die primär chirurgische Therapie ist für jene Patienten mit einer intraperitonealen Ruptur reserviert, welche einer kompletten Exploration und Lavage des Abdomens bedürfen. Weiterhin ist sie bei einer begleitenden intraabdominalen Erkrankung sowie bei Versagen der Behandlung durch PAD und Antibiotika indiziert. Es kann in diesen Fällen neben der konventionellen Laparotomie auch die Laparoskopie mit Drainageanlage effektiv und sicher durchgeführt werden. Eine Leberresektion ist selten notwendig, da in den meisten Fällen die o. g. Maßnahmen zur Abheilung führen.

\subsection{Leberversagen}

Das Leberversagen ist eine schwerwiegende Erkrankung mit der Entwicklung einer Leberfunktionsstörung, die mit einem Ikterus, metabolischer Entgleisung, Koagulopathie und Enzephalopathie einhergeht. Es wird im Hinblick auf das Auftreten des Ikterus und auf die Entwicklung einer Enzephalopathie in ein hyperakutes Leberversagen (Enzephalopathie 
- Tab. 21.5 Ursachen eines Leberversagens

Virale Infektionen

Medikamente und Toxine

Vaskulär

Metabolisch

Traumatisch und postoperativ

Sonstige

Unklare Ursache
Hepatitis-Virus A, B, C, D, E, Herpes simplex, Zytomegalievirus, Epstein-Barr-Virus, Herpes zoster, Adenovirus, hämorrhagisches Fieber

Acetaminophen, Amanita phalloides (Knollenblätterpilz), Ecstasy, Halothan, Isoniazid, Rifampicin, nichtsteroidale Antirheumatika, pflanzliche Heilmittel, Valproat

Rechtsherzversagen, Budd-Chiari-Syndrom, Venookklusive Erkrankung, ischämische Hepatitis, Hitzeschock

M. Wilson, Reye-Syndrom, HELLP-Syndrom, fatty liver of pregnancy (FLOP)

Leberruptur, primäres Transplantatversagen, postoperativ nach Leberresektion

Autoimmunhepatitis, Sepsis

- Tab. 21.6 Diagnostik beim Leberversagen

Anamnese und körperliche Untersuchung

Labormedizinische Untersuchungen

Mikrobiologie und Virologie

Weitere Diagnostik
Aktueller Krankheitsverlauf, Vorerkrankungen, Voroperationen, Medikamentenanamnese, Größe, Gewicht, Allgemein- und Ernährungszustand, Haut und Skleren, Neurologie, Thorax, Herz, Abdomen

Hämoglobin ( $\mathrm{Hb}$ ), Thrombozyten, Leukozyten, Quick bzw. INR, Blutgruppe, Glukose, Bilirubin, AlaninAminotransferase (ALAT), Aspartat-Aminotransferase (ASAT), alkalische Phosphatase (AP), Albumin, Elektrophorese, Immunglobuline, Kreatinin, Harnstoff, Elektrolyte im Serum und Urin, Blutgasanalyse, Drogenscreening, Kupfer im Urin, Coeruloplasmin

Hepatitis-B-Antigen und IgM-Anticore, Hepatitis-A-Antikörper (IgM), Hepatitis-C-Antikörper, Hepatitis-EAntikörper, Autoimmunantikörper, Blutkultur, Sputumkultur, Urinkultur, Stuhl auf pathogene Keime

Ultraschall des Abdomens, Röntgen-Thorax, Echokardiographie (EKG), Bilanzierung innerhalb von 7 Tagen nach Auftreten des Ikterus), ein akutes Leberversagen (Enzephalopathie innerhalb von 8-28 Tagen nach Auftreten des Ikterus) und ein subakutes Leberversagen (Enzephalopathie mehr als 28 Tage nach Auftreten des Ikterus) unterschieden. In - Tab. 21.5 werden die Ursachen eines Leberversagens dargestellt. Die akute Dekompensation einer chronischen Lebererkrankung, das sogenannte Acute-onchronic-Leberversagen, ist hiervon zu unterscheiden. Es tritt bei Patienten mit einer Zirrhose auf und wird häufig durch eine Infektion oder eine gastrointestinale Blutung verursacht. Weiterhin davon abzugrenzen ist die Entwicklung eines postoperativen Leberversagens als Komplikation nach einer Leberresektion oder anderen chirurgischen Eingriffen, insbesondere dann, wenn eine vorbestehende Schädigung der Leber und Resektion von großen Anteilen funktionierenden Leberparenchyms vorlagen.

\subsubsection{Klinik und Diagnostik}

Die Patienten stellen sich meistens einige Tage nach Beginn eines Ikterus vor. Es bestehen unspezifische Symptome wie ein allgemeines Krankheitsgefühl, Bauchschmerzen, Übelkeit, Erbrechen, Konzentrationsschwäche, Desorientierung und Verwirrtheit. Die Diagnose wird durch die Anamnese und die körperliche sowie labormedizinische Untersuchung gestellt (•Tab. 21.6). Das Leberversagen kann zu einem Syndrom mit den klinischen Merkmalen einer Oligurie, Azidose,
Laktatämie, Hypotension mit hyperdynamischer Zirkulation, Koagulopathie, Hypoglykämie, akutem Lungenversagen und ARDS (acute respiratory distress syndrome) führen. In vielen Fällen bleibt die Ursache des Leberversagens unklar.

\subsubsection{Therapie}

Die Behandlung eines Patienten mit einem Leberversagen erfolgt wegen des o. g. drohenden Multiorganversagens auf einer Intensivstation. Der Kontakt mit einem Leberzentrum ist dringend anzuraten, damit rechtzeitig mit der Abklärung der Diagnose, Einleitung der angemessenen Therapie und Verlegung in ein Transplantationszentrum begonnen werden kann.

Eine Lebertransplantation ist als definitive Behandlung eines Patienten mit einem Leberversagen dann indiziert, wenn die Wiederherstellung der Leberfunktion als unwahrscheinlich angesehen wird. Die Bundesärztekammer hat hierzu Kriterien in der Richtlinie gemäß $\$ 16$ Abs. 1 S. 1 Nrn. 2 u. 5 Transplantationsgesetz (TPG) für die Wartelistenführung und Organvermittlung zur Lebertransplantation veröffentlicht (-Tab. 21.7).

Die Standardtechnik der Lebertransplantation im eigenen Vorgehen erfolgt über eine rechtsseitige Oberbauchlaparotomie, welche median zum Xyphoid verlängert wird. Eine Erweiterung nach links ist in manchen Fällen notwendig. Beim akuten Leberversagen ist die Hepatektomie des Empfängers 


\begin{tabular}{|c|c|}
\hline $\begin{array}{l}\text { King's } \\
\text { College- } \\
\text { Kriterien }\end{array}$ & $\begin{array}{l}\text { Prothrombinzeit }>100 \mathrm{~s} \text { (= Quick }<7 \% \text { bzw. } \\
\text { INR }>6,7 \text { ) } \\
\text { oder mindestens } 3 \text { der folgenden: } \\
\text { - Ungünstige Ätiologie: kryptogene Hepatitis, } \\
\text { Halothan-Hepatitis, Medikamententoxizität } \\
\text { - Ikterus mehr als } 7 \text { Tage vor Enzephalopathie } \\
\text { - Alter }<10 \text { Jahre oder }>40 \text { Jahre } \\
\text { - Prothrombinzeit }>50 \mathrm{~s} \text { (= Quick }<15 \% \text { bzw. } \\
\text { INR }>4 \text { ) } \\
\text { - Serum-Bilirubin }>300 \mathrm{mmol} / /\end{array}$ \\
\hline $\begin{array}{l}\text { Kriterien für } \\
\text { die } \\
\text { Paracetamol- } \\
\text { intoxikation }\end{array}$ & $\begin{array}{l}\text { Arterieller } \mathrm{pH}<7,3 \\
\text { oder alle } 3 \text { folgenden: } \\
\text { - Prothrombinzeit }>100 \mathrm{~s} \text { (= Quick }<7 \% \text { bzw. } \\
\text { INR }>6,7 \text { ) } \\
\text { - Kreatinin }>300 \mathrm{mmol} / \mathrm{I} \\
\text { - Enzephalopathie Grad } 3 \text { oder } 4\end{array}$ \\
\hline $\begin{array}{l}\text { Kriterien für } \\
\text { eine Virus- } \\
\text { hepatitis } \\
\text { (Clichy- } \\
\text { Kriterien) }\end{array}$ & $\begin{array}{l}\text { Enzephalopathie Grad } 3 \text { und } 4 \\
\text { und } \\
\text { Faktor } V<20 \% \text { bei Empfängern }<30 \text { Jahre } \\
\text { oder } \\
\text { Faktor } V<30 \% \text { bei Empfängern }>30 \text { Jahre }\end{array}$ \\
\hline
\end{tabular}

wegen der fehlenden portalen Hypertension und venösen Umgehungskreisläufe relativ einfach und die Anlage eines veno-venösen Bypasses nicht zwingend erforderlich.

Die orthotope Transplantation kann in konventioneller Technik mit Anastomose der supra- und infrahepatischen V. cava oder in der Piggy-back-Technik, die den Erhalt der V. cava inferior des Empfängers erfordert, durchgeführt werden. In diesem Fall wird die Anastomose mit der suprahepatischen V. cava des Spenderorgans und den Lebervenen des Empfängers durchgeführt. Es erfolgt dann die portale Anastomose und in aller Regel danach die Reperfusion des Organs. Nach der Reperfusion wird die arterielle Anastomose zwischen der A. hepatica des Spenders und Empfängers durchgeführt. Abschließend erfolgt die Wiederherstellung der ableitenden Gallenwege, welche als Choledocho-Choledochostomie bzw. Hepatico-Hepaticostomie oder als Hepatojejunostomie rekonstruiert werden.

Ergänzend sei auf die Alternativen zur Standardtechnik hingewiesen, die jedoch nur einen geringen Anteil bei der chi- rurgischen Behandlung des Leberversagens haben: die partielle Lebertransplantation, die Splitlebertransplantation, die Leberlebendspende und die auxilliäre Lebertransplantation.

Die Spenderleber wird bei der partiellen Lebertransplantation segmentorientiert, meistens Segment II-III, größenreduziert und in einen kleinen Empfänger orthotop transplantiert. Die Splitlebertransplantation ermöglicht die Transplantation von 2 Empfängern mit einem Spenderorgan. Das Splitting kann während der Multiorganentnahme als In-situ-Split oder nach der Organentnahme als Ex-situ-Split erfolgen. Die Leberlebendspende erfolgt unter Verwendung des rechten, linken oder links-lateralen Leberanteils eines gesunden Spenders. Dieser Leberanteil wird orthotop in der oben beschriebenen Piggy-back-Technik transplantiert. Die zusätzliche Transplantation einer Spenderleber oder eines Spenderleberteils unter Belassung der empfängereigenen Leber wird als auxilliäre Lebertransplantation bezeichnet und soll die Erholung der geschädigten Leber beim akuten Leberversagen ohne Notwendigkeit einer lebenslangen Immunsuppression ermöglichen.

\subsubsection{Praktische Hinweise}

- Die Diagnose eines Leberversagens kann häufig durch die Anamnese, klinische Untersuchung und Interpretation der labormedizinischen Untersuchungen relativ schnell erfolgen.

- Die Beseitigung der Ursache (falls möglich) sowie intensivmedizinische und supportive Maßnahmen sind die tragenden Säulen in der Behandlung des Leberversagens.

- Das Leberversagen ist eine schwere Erkrankung, die tödlich verlaufen kann. Patienten mit der Trias Enzephalopathie, Koagulopathie und metabolischer Entgleisung sollten daher unverzüglich in einem Lebertransplantationszentrum vorgestellt bzw. dorthin verlegt werden.

- Die Wahrscheinlichkeit der Wiederherstellung der Leberfunktion wird entsprechend der zugrunde liegenden Ursache anhand der King's College-Kriterien, Kriterien für die Paracetamolintoxikation oder der Clichy-Kriterien für Virushepatitiden abgeschätzt. Diese Kriterien können bei der Selektion der Patienten hilfreich sein, die von einer Lebertransplantation profitieren. 


\subsection{Komplikationen nach ERCP und interventionellen Eingriffen}

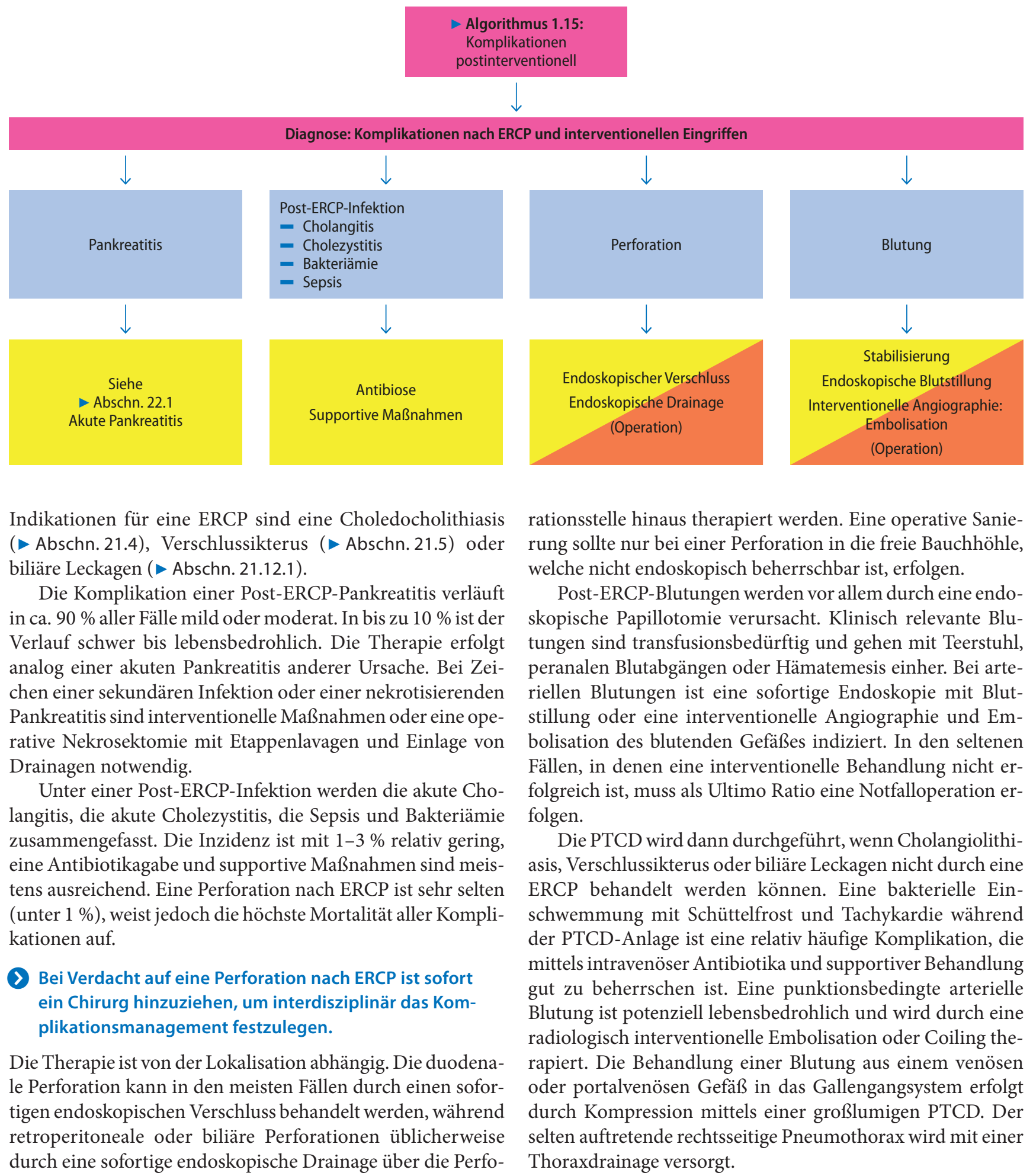




\subsection{Postoperative Komplikationen}

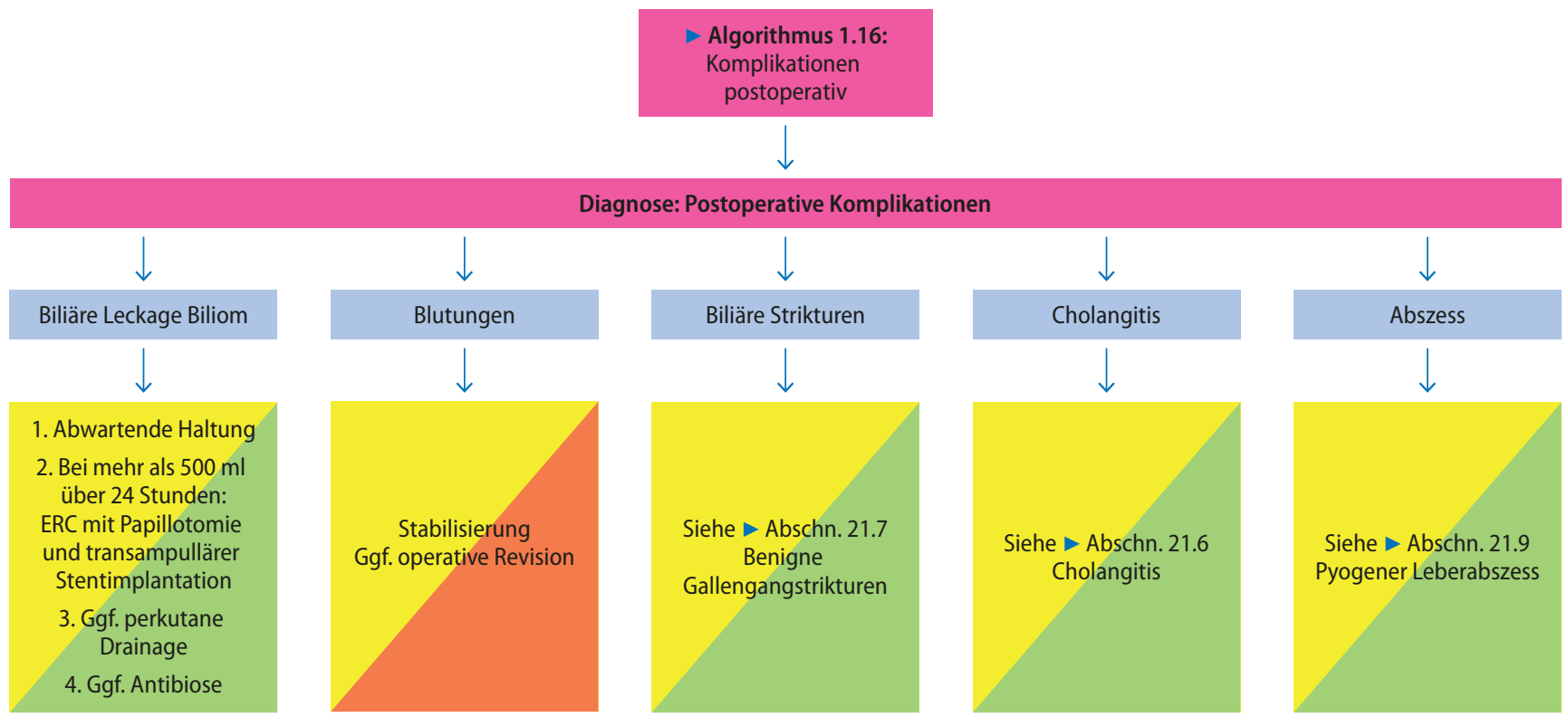

Beschwerden nach hepatobiliären Operationen bedürfen einer weiterführenden Diagnostik mittels Sonographie, CT, MRT/MRCP, ERC. Die häufigsten postoperativen Komplikationen nach einer Operation an der Gallenblase, an den Gallewegen und an der Leber sind biliäre Leckagen, Biliome, Abszessbildung, Blutungen, Hämatome, biliäre Stenosen und Strikturen verbunden mit einer Cholangitis. Daneben können, insbesondere nach einer erweiterten Leberresektion, ein Pleuraerguss und eine postoperative Leberinsuffizienz auftreten.

Postoperative Flüssigkeitsansammlungen wie Biliom, Hämatom oder Abszess können mit Sonographie, CT und MRT/MRCP festgestellt werden. Eine akute und subakute Blutung zeigt gewöhnlich eine höhere Dichte im CT und kann daher zuverlässig diagnostiziert werden. Zusätzlich kann eine Blutung als Kontrastmittelaustritt aus einem Gefäß mit einem kontrastmittelverstärkten CT bewiesen werden. Ein Biliom zeigt typischerweise in der Bildgebung einen an die Resektionsfläche angrenzenden abgekapselten raumfordernden Prozess.

\subsubsection{Biliäre Leckage}

Biliäre Leckagen nach Gallenblasenoperationen aus dem Gallenblasenbett entstehen häufig durch eine zu tiefe Präparationsebene in das Leberparenchym hinein. Weitere Ursachen sind die Durchtrennung eines akzessorischen Gallengangs, eine Zystikusstumpfinsuffizienz oder eine Verletzung des DHC.

\section{Praxistipp}

Akzessorische Gallengänge und biliäre Leckagen, sofern sie während der Präparation im Gallenblasenbett identifiziert werden, sollen unbedingt ligiert oder besser durchstochen werden, da diese zu postoperativen Biliomen und sekundär zu Abszessen führen können.

Eine biliäre Leckage kann dann toleriert werden, sofern keine Zeichen einer galligen Peritonitis oder einer Sepsis bestehen. Kontrollen der Sekretion aus einliegenden intraabdominalen Drainagen sollten daher engmaschig erfolgen. Im Falle einer geringen galligen Sekretion und fehlender klinischer Symptome sowie einer ausreichenden Drainage ist eine abwartende Haltung gerechtfertigt. Eine persistierende Leckage von mehr als $500 \mathrm{ml}$ über 24 Stunden sollte mittels ERC mit Papillotomie und transampullärer Stentimplantation in den DHC behandelt werden. Es kommt hierdurch zu einer Dekompression des Gallenwegsystems und der Gallenfluss wird vom Defekt in die Gallenwege umgeleitet.

Biliäre Leckagen nach Leberresektionen entstehen häufig aufgrund sekundärer Nekrosen am Resektionsrand (- Abb. 21.10).

( ) Tiefgreifende Nähte sind daher wegen des hohen Risikos, wesentliche Gefäß- oder Gallengangstrukturen intrahepatisch mitzufassen und Nekrosen des Leberparenchyms zu provozieren, unbedingt zu vermeiden.

Eine Vielzahl dieser biliärer Leckagen heilen spontan. Im Falle einer persistierenden biliären Leckage ist mit einem verlängerten stationären Aufenthalt zu rechnen und eine Entlastung des Gallengangsystems durch eine ERC mit Papillotomie 


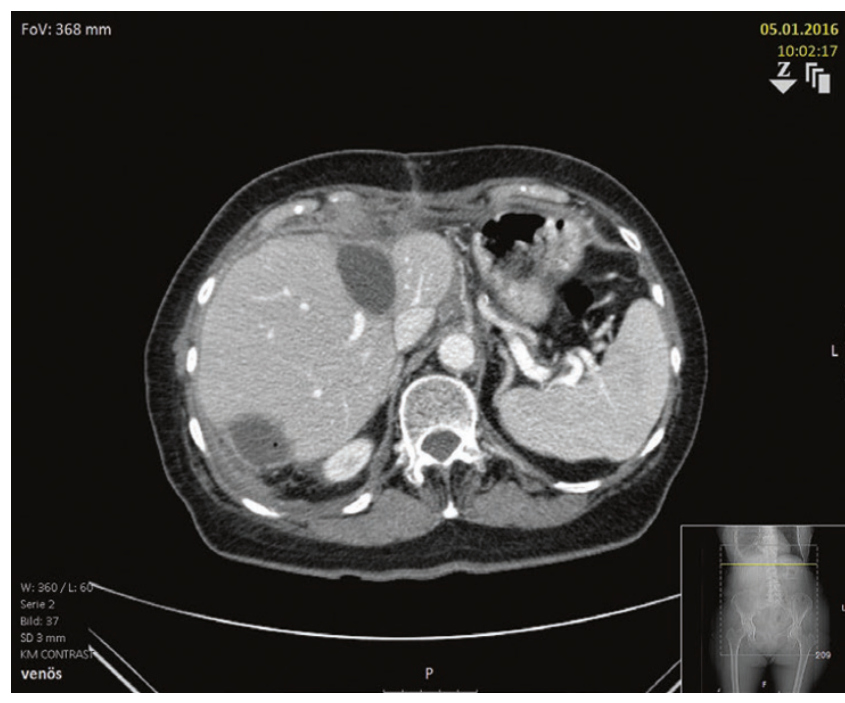

- Abb. 21.10 Postoperative Biliome am Resektionsrand nach Resektion kolorektaler Lebermetastasen

und transampullärer Stentimplantation in den DHC oder eine PTCD anzustreben. Die Indikation zur Revisionsoperation nach Leberresektion sollte bei ausgedehnten Gallefisteln und entsprechender Symptomatik gestellt werden.

\section{Praxistipp}

In der frühpostoperativen Phase nach Leberresektion ist ein Übernähen einer biliären Leckage meistens noch möglich. Besteht eine Leckage bereits länger als 8 Tage, ist wegen der entzündlichen Veränderungen ein sicherer Verschluss wesentlich schwieriger zu erreichen.

\subsubsection{Biliäre Strikturen}

Siehe dazu $>$ Abschn. 21.7 und $\bullet$ Abb. 21.11.

\subsubsection{Blutungen}

Blutungen während oder nach Gallenblasenoperationen, im Besonderen bei Patienten mit einer zugrunde liegenden akuten Cholezystitis oder einer Zirrhose, entstehen im Allgemeinen am Gallenblasenbett. Eine zu tiefe Präparationsebene in das Leberparenchym hinein kann zu einer Blutung führen. Weitere Ursachen sind eine Verletzung oder Durchtrennung der A. cystica, eine akzidentelle Verletzung der Leberarterie sowie ein Abgleiten der Ligatur bzw. der Endoclips. Eine weitere Blutungsursache können die Trokarstellen darstellen.

Das Management einer Blutung während der laparoskopischen Cholezystektomie kann risikoreich und mit erheblichen Auswirkungen verbunden sein. Dieses beinhaltet Ligaturen oder Verletzungen größerer Gefäße und/oder der

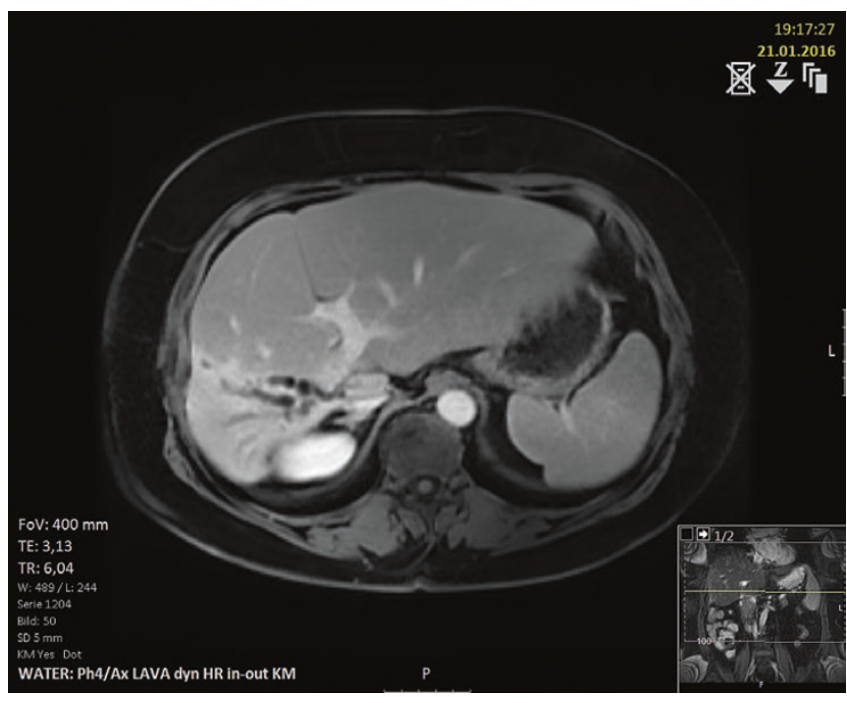

- Abb. 21.11 Cholestase des rechten Leberlappens aufgrund einer langanhaltenden Obstruktion mit chronischer Cholangitis, konsekutiver biliärer Zirrhose, sekundärer Leberatrophie und Hypertrophie der Lebersegmente IVa und IVb

Gallenwege durch ungezielte Clipapplikation oder Gebrauch des Elektrokauters in einem schlecht visualisierten Operationsfeld. In solchen Fällen ist die Konversion zu einer Laparotomie das oftmals weitaus bessere Vorgehen, um eine gezielte Blutstillung zu erzielen.

\subsubsection{Cholangitis}

Das Erscheinungsbild und der Verlauf einer Cholangitis ( $\downarrow$ Abschn. 21.6) reicht von leichten, in Schüben verlaufenden und rezidivierenden Episoden von Abdominalschmerzen, Ikterus, Fieber und Schüttelfrost bis hin zu einer schnell fortschreitenden systemischen Erkrankung, die zu Schock, Sepsis, Leberkoma und Tod führen kann. Die Entwicklung einer Cholangitis erfordert 3 wesentliche Faktoren:

1. Obstruktion des Gallenflusses

2. Kolonisation der Gallenflüssigkeit mit Bakterien und Pilze

3. Erhöhung des intraduktalen Galledrucks

Postoperative Strikturen treten häufig nach Cholezystektomien mit intraoperativ nicht erkannten Schädigungen des DHC und nach Gallenwegsoperationen mit Anlagen von biliodigestiven Anastomosen auf.

Zur Therapie siehe $>$ Abschn. 21.6.2 und $\bullet$ Abb. 21.12.

\subsubsection{Abszess}

Die häufigsten Ursachen von Abszessbildungen sind Galleleckagen nach Gallenblasen- bzw. Gallenwegoperationen und Leberresektionen.

Zur Therapie siehe Abschn. 21.9.2. 


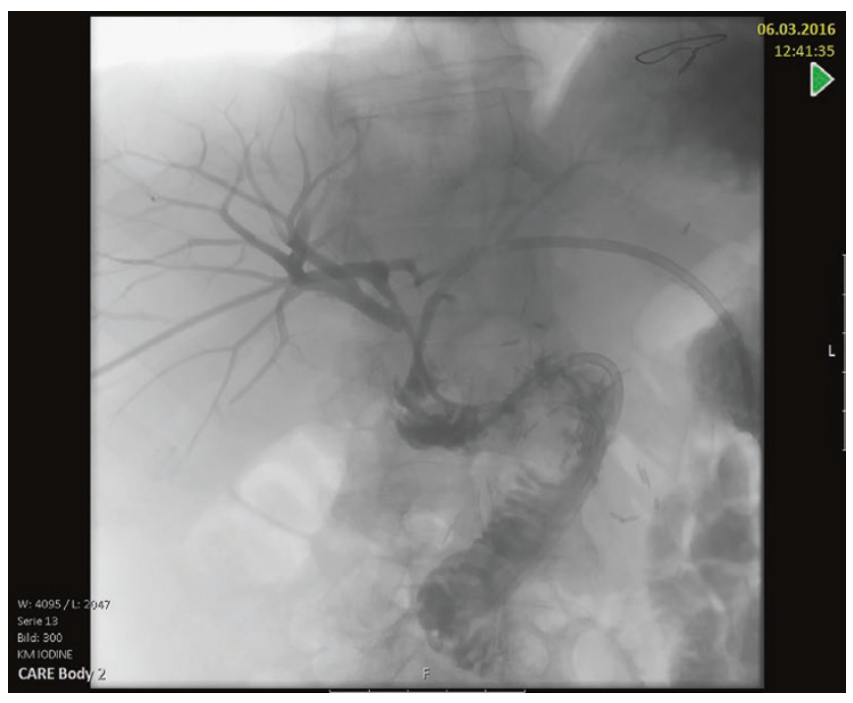

- Abb. 21.12 PTCD-Anlage im Rahmen einer Cholangitis aufgrund einer Striktur der biliodigestiven Anastomose nach pyloruserhaltender partieller Pankreatoduodenektomie

\subsubsection{Biliom}

Das Biliom ist eine abgekapselte Kollektion von Gallenflüssigkeit innerhalb des Abdomens und wird als postoperative Komplikation nach einer Operationen an Gallenblase, Gallenwegen, Leberresektionen oder Lebertransplantationen beobachtet. Ursache ist eine biliäre Leckage nach Verletzung an den Gallenwegen, Zystikusstumpfinsuffizienz, Anastomoseninsuffizienz nach Anlage einer biliodigestiven Anastomose oder einer termino-terminalen Gallenwegsanastomose, z. B. im Rahmen einer Lebertransplantation.

Die Diagnose wird bildgebend mittels Sonographie, CT oder MRT gestellt.

Die Therapie eines Bilioms erfolgt in Abhängigkeit der klinischen Beschwerden, der Größe, der Lokalisation und der Ursache. Eine Zystikusstumpfinsuffizienz und Verletzungen des DHC mit biliären Leckagen werden mittels ERC überstentet. Der Stent sollte mindestens 14 Tage belassen und nach Ausheilung der Insuffizienz entfernt werden. Bei klinisch inapparenten kleinen Biliomen ist ein abwartendes Vorgehen gerechtfertigt. Eine perkutane Drainage und antibiotische Behandlung ist bei einem größeren Biliom mit begleitender Infektion indiziert (• Abb. 21.13).

\section{Literatur}

Anderson MA et al. (2012) ASGE Standards of Practice Committee: Complications of ERCP. Gastrointest Endosc 75(3): 467-473

Arend J et al. (2015). Gallenleckage nach Leberresektion. Der Chirurg $86: 132-138$

Clarke DL et al. (2006) The current standard of care in the periprocedural management of the patient with obstructive jaundice. Ann R Coll Surg Eng 88: 610-616

Crespi M et al. (2016) Diagnosis and Treatment of Biliary Fistulas in the Laparoscopic Era. Gastroenterol Res Pract. 2016:6293538. doi: 10.1155/2016/6293538. Epub 2015 Dec 24

Drapanas T (1972) Interposition mesocaval shunt for treatment of portal hypertension. Ann Surg 176:435-448

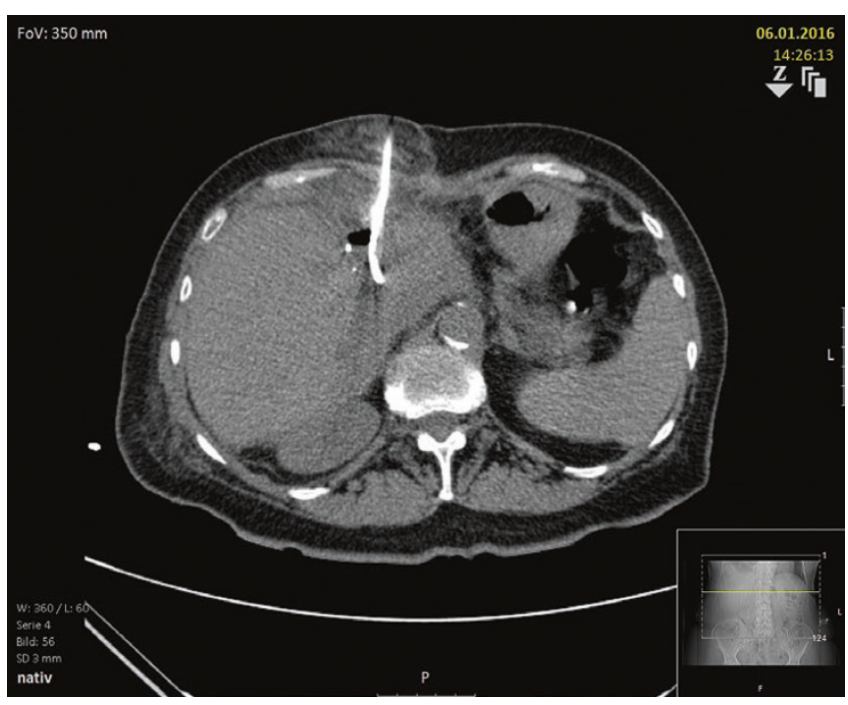

- Abb. 21.13 Behandlung eines infizierten Bilioms mit einer perkutanen Drainage

Duncan CB, Riall TS (2012) Evidence-Based Current Surgical Practice: Calculous Gallbladder Disease J Gastrointest Surg 16:2011-2025 Götzky K et al. (2013) Epidemiologie und Klinik der akuten Cholezystitis. Chirurg 84:179-184

Gutt CN et al. (2013) Acute cholecystitis: early versus delayed cholecystectomy, a multicenter randomized trial (ACDC study, NCT00447304). Ann Surg 258(3):385-393

Hartwig W et al.(2013) Minimal-invasive chirurgische Therapie der akuten Cholezystitis. Der Chirurg 84:191-196

Lammert F et al. (2007) S3-Leitlinie der Deutschen Gesellschaft für Verdauungs- und Stoffwechselkrankheit und der Deutschen Gesellschaft für Viszeralchirurgie zur Diagnostik und Behandlung von Gallensteinen. AWMF -Register-Nr. 21/008. Z Gastroenterol 45: 971-1001

Mendizabal M, Silva MO (2016) Liver transplantation in acute liver failure: A challenging scenario. World J Gastroenterol 22(4):1523-1531

Qualitätsreport des Gemeinsamen Bundesausschuss 2014. https://www. aqua-institut.de/de/aktuelles/qualitaetsreport-2014.html. Zugegriffen: 29. März 2016

Nagel E, Löhlein D (2006) Pichlmayers Chirurgische Therapie. Berlin: Springer

Rabenstein T, Schulz HJ (2014) Komplikationen nach ERCP. Der Gastroenterologe 9: 222-236

Radeleff BA et al. (2005) Interventionen bei malignen Gallenwegstenosen. Der Radiologe 45:1020-1030

Schima W et al.2010 Erkrankungen des hepatobiliären Systems als Ursache des akuten Abdomens. Der Radiologe 50:252-261

Wang-Chan A et al. (2014) Abdominalbeschwerden nach Gallensteinileusoperation. Der Chirurg 85:342-344

\section{Lehrbücher}

Jarnagin WR et al. (2012) Blumgart's Surgery of the Liver, Biliary Tract and Pancreas, 5. Aufl. Amsterdam: Elsevier

Korenkov M, Germer CT, Lang H (Hrsg) (2013) Gastrointestinale Operationen und technische Varianten. Berlin: Springer

Dooley JS, Lok A, Burroughs AK, Heathcote J (Hrsg) (2011) Sherlock's Diseases of the Liver and Biliary System, 12. Aufl. Hoboken/New Jersey: Wiley-Blackwell

Siewert JR, Schumpelick V, Rothmund M (Hrsg) (2011) Praxis der Viszeralchirurgie: Gastroenterologische Chirurgie. Berlin: Springer

Carter, Russel, Pitt, Bismuth (1996) Rob \& Smith's Operative Surgery. Hepatobiliary and Pancreatic Surgery, 5. Aufl. Chapman \& Hall Medical 